\title{
Current-Density Functional Theory for the superconductor
}

\author{
Katsuhiko Higuchi \\ Graduate School of Advanced Sciences of Matter, \\ Hiroshima University, Higashi-Hiroshima 739-8527, Japan \\ Masahiko Higuchi \\ Department of Physics, Faculty of Science, \\ Shinshu University, Matsumoto 390-8621, Japan
}

(Dated: October 4, 2018)

\begin{abstract}
We present the current-density functional theory for the superconductor immersed in the magnetic field. The order parameter of the superconducting state, transverse component of the paramagnetic current-density, and electron density are chosen as basic variables that uniquely determine the equilibrium properties of the system. In order to construct this theory, the development of the approximate form of the exchange-correlation (xc) energy functional is indispensable as well as the derivation of the effective single-particle equation which makes it possible to reproduce the equilibrium densities mentioned above. The rigorous expression of the xc-energy functional is derived using the technique of the coupling-constant integration. Furthermore, the approximate form of the xc energy functional is proposed such that the energy gap resulting from the effective single-particle equation is consistent with the attractive interaction energy of the system.
\end{abstract}

PACS numbers: 74.20.Pq

Keywords: current-density functional theory, order parameter, superconductivity, paramagnetic currentdensity, exchange-correlation energy functional, critical magnetic field, critical temperature, critical currentdensity 


\section{INTRODUCTION}

The superconductivity has been one of the main topics in the condensed matter physics. In order to clarify the properties of the superconducting state, the first-principles approaches which can evaluate the order parameter of the superconducting state (OPSS) quantitatively play an important role $\mathrm{e}^{\underline{-21}}$ as well as the other approaches using the model Hamiltonian 22 . One of the most popular first-principles theories is the density functional

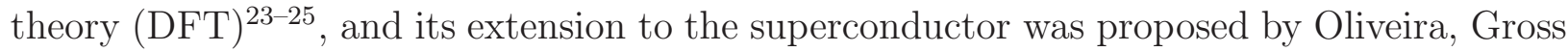
and Kohn $(\mathrm{OGK})^{26,27}$. Since it can predict not only the critical temperature of the superconductivity but also the properties of the OPSS, i.e., spatial distribution and spin symmetry for the OPSS, the OGK theory has been applied to a lot of superconductors $1-21,23-27$.

However, the OGK theory cannot in principle predict the critical current-density because the current-density is not chosen as a basic variable to be reproduced 26 . From the viewpoint of the practical applications of superconductors, the critical current-density is also an important quantity as well as the critical temperature 28,29 . The critical current-density is related to the critical magnetic field via the London equation, which is so-called Silsbee's rule $30,31$. The larger the critical current-density is, the larger the critical magnetic field is ${ }^{30,31}$. Therefore, if the critical current-density is needed, we have only to calculate the critical magnetic field.

In order to develop the first-principles theory for calculating the critical magnetic field, we need to deal with the superconductor immersed in the external magnetic field. Specifically, we need to construct the current-density functional theory (CDFT) for the superconductor, in which the dependence of the OPSS on the external magnetic field can be predicted. We shall stress once again that it is necessary to construct the CDFT for the superconductor so as to predict the critical current-density that is a key quantity for the practical use of the superconductor.

The CDFT for the solids of the normal state has previously been proposed by Vignale and Rasolt ${ }^{32,33}$, and on the basis of this theory, the CDFT for the superconductor has been developed by Kohn, Oliveira and Gross (KOG) ${ }^{27}$. However, unfortunately, the KOG theory may be regarded as a prototype of the CDFT for the superconductor, and it should be improved and extended in the following points:

(i) The OPSS is essentially the two-variable function concerning both spin and spatial 
coordinates, because the OPSS contains as a part the two-particle wave function resulting from the Bose-Einstein condensation (BEC) of the fermion system 34 , 35 . In the previous work $^{27}$, the OPSS was treated only in the restricted form with the spatially local and spinsinglet one. This restricted form cannot describe the center of the gravity of two particles that form the Cooper pair, so that the vortex pattern of the mixed state of the type II superconductors cannot be described. The spatial and spin dependences of the OPSS should be treated as they are without any approximation.

(ii) In the proof of the Hohenberg-Kohn (HK) theorem which will be shown in Sec. 3, the technique of the constrained-search $\underline{36} \underline{\underline{43}}$ is used to avoid the difficulty of the assumption of the $v$-representability $27, \underline{44}$. Specifically, we will extend the extended constrained-search (ECS) theory $\underline{39-43}$ to the case of the finite temperature ${ }^{25}$, and apply it to the CDFT for the superconductor.

(iii) In the conventional CDFT, the electron density and paramagnetic current-density have been adopted as the basic variables that uniquely determine the ground-state properties or equilibrium properties of the system $27,32,33, \underline{45}-\underline{47}$. However, they are not independent of each other. Specifically, the electron density and the longitudinal component of the paramagnetic current-density are related to each other via the equation of continuity. When performing the variational principle with respect to the basic variables, it is more convenient to choose as the basic variables the physical quantities that are independent of each other. Therefore, in this paper, the transverse component of the paramagnetic current-density is chosen as one of the basic variables instead of the whole components of the paramagnetic current-density.

(iv) In the development of the DFT-based theory or ECS-based theory, the following two issues are indispensable and are closely connected with each other. One is to derive the effective single-particle equation on the basis of the HK theorem, which is so-called the

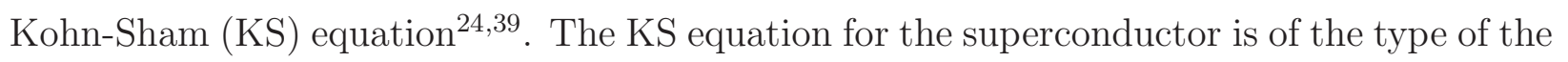
Bogoliubov-de Gennes (BdG) equation플, which is hereafter called BdG-KS equation. The other is to develop the approximate form of the exchange-correlation (xc) energy functional that is contained in the BdG-KS equation. The former issue just corresponds to the abovementioned (ii) and (iii). As for the latter issue, there have been no attempts in the previous $\operatorname{work}^{27}$. In this paper, we will derive the rigorous expression for the xc energy functional, and present the approximate form which is consistent with the attractive interaction between 
electrons.

In this paper, we present the CDFT for the superconductor with particular emphasize on the above-mentioned four points. Especially, (iv) is the most essential point of whether the present theory works well or not. Organization of this paper is as follows. In Sec. II, the OPSS is reviewed on the basis of the definition of the superconductivity $\underline{34}$. In Sec. III, the ECS theory ${ }^{39-43}$ is extended to the case of the finite temperature. The HK theorem is proven without the assumption of the $v$-representability. Introducing the reference system in which the HK theorem holds, the BdG-KS equation is derived in the case of the finite temperature in Sec. IV. In Sec. V, the effective mean-field potentials that are contained in the BdG-KS equation are determined by requiring that the transverse component of the paramagnetic current-density, electron density and OPSS of the equilibrium state are reproduced in the reference system. The development of the xc energy functional is one of main topics in the present paper. Not only the rigorous expression but also the approximate forms of the xc energy functional are presented in Sec. VI. Finally, some concluding remarks are given in Sec. VII.

\section{ORDER PARAMETER OF THE SUPERCONDUCTING STATE}

The superconducting state can be regarded as the BEC of the fermion system $\stackrel{34}{ }$. The BEC of the fermion system is defined by using the second-order reduced density matrix (RDM2) ${ }^{34,35}$. According to this definition, when the BEC occurs in the system, one of eigenvalues for the RDM2 takes the value of $O(N)$ and the corresponding eigenfunction extends in terms of the coordinate of the center of the gravity but localizes in terms of the relative coordinate ${ }^{49}$. In that case, it is shown that the following quantity takes the nonzero value $\underline{\underline{50}-52}$ :

$$
\Delta\left(\mathbf{r}_{2} \zeta_{2}, \mathbf{r}_{1} \zeta_{1}\right)=\left\langle\hat{\Delta}\left(\mathbf{r}_{2} \zeta_{2}, \mathbf{r}_{1} \zeta_{1}\right)\right\rangle \quad \text { with } \quad \hat{\Delta}\left(\mathbf{r}_{2} \zeta_{2}, \mathbf{r}_{1} \zeta_{1}\right)=\psi\left(\mathbf{r}_{1} \zeta_{1}\right) \psi\left(\mathbf{r}_{2} \zeta_{2}\right)
$$

where $\psi(\mathbf{r} \zeta)$ is the field operator of electrons, and where $\mathbf{r}$ and $\zeta$ are spatial and spin coordinates, respectively. The bracket in the RHS means the expectation value with respect to the ground state in the case of zero temperature or the statistical average in the case of the finite temperature. $\Delta\left(\mathbf{r}_{2} \zeta_{2}, \mathbf{r}_{1} \zeta_{1}\right)$ is exactly the OPSS, and is coincident with the square root of the maximum eigenvalue multiplied by the corresponding eigenfunction for 
the RDM2 $2^{34,50}$.

Using the spin-polarized states $\varphi_{k_{i} \sigma_{i}}(\mathbf{r}) \chi_{\sigma_{i}}(\zeta)$, and the corresponding annihilation operator $C_{k_{i} \sigma_{i}}$, the field operator is rewritten as

$$
\psi(\mathbf{r} \zeta)=\sum_{k_{i}} \sum_{\sigma_{i}=\uparrow, \downarrow} \varphi_{k_{i} \sigma_{i}}(\mathbf{r}) \chi_{\sigma_{i}}(\zeta) C_{k_{i} \sigma_{i}}
$$

where $\chi_{\sigma_{i}}(\zeta)$ is the spin function. Substituting Eq. (2) into Eq. (1), we have

$$
\begin{aligned}
& \Delta\left(\mathbf{r}_{2} \zeta_{2}, \mathbf{r}_{1} \zeta_{1}\right)=\frac{1}{2} \sum_{k_{i}} \sum_{k_{j}}\left\langle C_{k_{i} \uparrow} C_{k_{j} \uparrow}\right\rangle\left|\begin{array}{cc}
\varphi_{k_{i} \uparrow}\left(\mathbf{r}_{1}\right) & \varphi_{k_{j} \uparrow}\left(\mathbf{r}_{1}\right) \\
\varphi_{k_{i} \uparrow}\left(\mathbf{r}_{2}\right) & \varphi_{k_{j} \uparrow}\left(\mathbf{r}_{2}\right)
\end{array}\right| \chi_{\uparrow}\left(\zeta_{1}\right) \chi_{\uparrow}\left(\zeta_{2}\right) \\
& +\frac{1}{2} \sum_{k_{i}} \sum_{k_{j}}\left\langle C_{k_{i \downarrow}} C_{k_{j} \downarrow}\right\rangle\left|\begin{array}{ll}
\varphi_{k_{i} \downarrow}\left(\mathbf{r}_{1}\right) & \varphi_{k_{j} \downarrow}\left(\mathbf{r}_{1}\right) \\
\varphi_{k_{i} \downarrow}\left(\mathbf{r}_{2}\right) & \varphi_{k_{j} \downarrow}\left(\mathbf{r}_{2}\right)
\end{array}\right| \chi_{\downarrow}\left(\zeta_{1}\right) \chi_{\downarrow}\left(\zeta_{2}\right) \\
& +\frac{1}{2} \sum_{k_{i}} \sum_{k_{j}}\left\langle C_{k_{i} \uparrow} C_{k_{j \downarrow}}\right\rangle\left|\begin{array}{cc}
\varphi_{k_{i} \uparrow}\left(\mathbf{r}_{1}\right) & \varphi_{k_{j} \downarrow}\left(\mathbf{r}_{1}\right) \\
\varphi_{k_{i} \uparrow}\left(\mathbf{r}_{2}\right) & \varphi_{k_{j} \downarrow}\left(\mathbf{r}_{2}\right)
\end{array}\right|\left\{\chi_{\uparrow}\left(\zeta_{1}\right) \chi_{\downarrow}\left(\zeta_{2}\right)+\chi_{\downarrow}\left(\zeta_{1}\right) \chi_{\uparrow}\left(\zeta_{2}\right)\right\} \\
& +\frac{1}{2} \sum_{k_{i}} \sum_{k_{j}}\left\langle C_{k_{i} \uparrow} C_{k_{j} \downarrow}\right\rangle\left\{\varphi_{k_{i} \uparrow}\left(\mathbf{r}_{1}\right) \varphi_{k_{j} \downarrow}\left(\mathbf{r}_{2}\right)+\varphi_{k_{j} \downarrow}\left(\mathbf{r}_{1}\right) \varphi_{k_{i} \uparrow}\left(\mathbf{r}_{2}\right)\right\}\left|\begin{array}{ll}
\chi_{\uparrow}\left(\zeta_{1}\right) & \chi_{\downarrow}\left(\zeta_{1}\right) \\
\chi_{\uparrow}\left(\zeta_{2}\right) & \chi_{\downarrow}\left(\zeta_{2}\right)
\end{array}\right|
\end{aligned}
$$

The first three terms of Eq. (3) are spin-triplet wave functions, and the fourth one is the spin-singlet wave function. Taking into account the facts that the eigenfunction and eigenvalue for the RDM2 mean the two-particle wave function and its occupation number in the BEC state, respectively $\underline{35}, \underline{50}$, it is recognized that the OPSS reflects the spin and spatial symmetries of the pairing state in the superconductor. Therefore, it is possible to find which type of the superconducting state, e.g., spin-singlet or spin-triplet or their mixed one, occurs by checking the OPSS.

Using the coordinate of center of gravity $\mathbf{R}$ and relative coordinate $\boldsymbol{\rho}$, where $\mathbf{R}=$ $\left(\mathbf{r}_{1}+\mathbf{r}_{2}\right) / 2, \boldsymbol{\rho}=\mathbf{r}_{1}-\mathbf{r}_{2}$, the OPSS is rewritten as $\Delta\left(\mathbf{R} \boldsymbol{\rho} ; \zeta_{1} \zeta_{2}\right)$ instead of $\Delta\left(\mathbf{r}_{2} \zeta_{2}, \mathbf{r}_{1} \zeta_{1}\right)$. By observing the dependence of $\Delta\left(\mathbf{R} \boldsymbol{\rho} ; \zeta_{1} \zeta_{2}\right)$ on $\boldsymbol{\rho}$, we can find the spatial broadening of the pairing states in the superconductor ${ }^{50}$. This enables us to discuss how close the pairing two electrons are to the Bose particle, which would characterize the properties of the superconducting state. Furthermore, by observing the dependence of $\Delta\left(\mathbf{R} \boldsymbol{\rho} ; \zeta_{1} \zeta_{2}\right)$ on $\mathbf{R}$, spatial distribution pattern of the OPSS can be found explicitly, which would also characterize the superconducting state. For example, in the type II superconductor, the magnetic flux periodically penetrates the portion of the superconductor in which the OPSS disappears. The dependence of $\Delta\left(\mathbf{R} \boldsymbol{\rho} ; \zeta_{1} \zeta_{2}\right)$ on $\mathbf{R}$ shows the spatial structure and pattern of the 
magnetic flux directly. Thus, the OPSS should be expressed in the form of possessing both dependences of $\boldsymbol{\rho}$ and $\mathbf{R}^{35,50}$.

\section{HOHENBERG-KOHN THEOREM}

In the previous work ${ }^{27}$, as mentioned in Sec. I, the $v$-representability of basic variables has been assumed in the proof of the HK theorem. The $v$-representability means the assumption where basic variables that determine the properties of the equilibrium state of the system have necessarily the corresponding external potentials $\stackrel{44}{*}$. In this section, we present the HK theorem of the CDFT for the superconductor without this assumption. Specifically, we construct the finite-temperature version of the ECS theory $\underline{39} \underline{43}$ that is suitable for the superconductor immersed in the external magnetic field.

\section{A. Hamiltonian}

Let us start with the Hamiltonian of the superconductor which is immersed in the beforehand-given electromagnetic fields $\mathbf{A}_{\text {given }}(\mathbf{r})$ and $v_{\text {given }}(\mathbf{r})$. These fields should be determined in a self-consistent way to express the Meissner effect 27 , which will be explained in the Sec. V. D. The Hamiltonian includes not only the Coulomb repulsive interaction but also the attractive interaction induced by some elementary excitation such as phonon. It is given by

$$
\begin{aligned}
\hat{H} & =\int \psi^{\dagger}(\mathbf{r} \zeta)\left[\frac{1}{2 m}\left\{\mathbf{p}+e \mathbf{A}_{\text {given }}(\mathbf{r})\right\}^{2}\right] \psi(\mathbf{r} \zeta) d^{3} r d \zeta \\
& +\frac{1}{2} \iint \psi^{\dagger}(\mathbf{r} \zeta) \psi^{\dagger}\left(\mathbf{r}^{\prime} \zeta^{\prime}\right) \frac{e^{2}}{\left|\mathbf{r}-\mathbf{r}^{\prime}\right|} \psi\left(\mathbf{r}^{\prime} \zeta^{\prime}\right) \psi(\mathbf{r} \zeta) d^{3} r d \zeta d^{3} r^{\prime} d \zeta^{\prime} \\
& +\frac{1}{2} \iint \psi^{\dagger}\left(\mathbf{r}_{1} \zeta_{1}\right) \psi^{\dagger}\left(\mathbf{r}_{2} \zeta_{2}\right) w\left(\mathbf{r}_{1} \zeta_{1} \mathbf{r}_{2} \zeta_{2} ; \mathbf{r}_{3} \zeta_{3} \mathbf{r}_{4} \zeta_{4}\right) \psi\left(\mathbf{r}_{3} \zeta_{3}\right) \psi\left(\mathbf{r}_{4} \zeta_{4}\right) d^{3} r_{1} d \zeta_{1} d^{3} r_{2} d \zeta_{2} d^{3} r_{3} d \zeta_{3} d^{3} r_{4} d \zeta_{4} \\
& +\int v_{\text {given }}(\mathbf{r}) \psi^{\dagger}(\mathbf{r} \zeta) \psi(\mathbf{r} \zeta) d^{3} r d \zeta .
\end{aligned}
$$

The second and third terms of the RHS denote the Coulomb repulsive interaction energy and attractive interaction energy via $w\left(\mathbf{r}_{1} \zeta_{1} \mathbf{r}_{2} \zeta_{2} ; \mathbf{r}_{3} \zeta_{3} \mathbf{r}_{4} \zeta_{4}\right)$, respectively. Neglecting the surface integral at infinite distance in a usual way $\underline{32,33,45,46}$, and adopting the Coulomb gauge given by $\nabla \cdot \mathbf{A}_{\text {given }}(\mathbf{r})=0$, the Hamiltonian is rewritten as

$$
\hat{H}=\hat{T}+\hat{W}_{1}+\hat{W}_{2}+\hat{V}_{1}+\hat{V}_{2}+\hat{V}_{3}
$$


with

$$
\begin{aligned}
& \hat{T}=\int \psi^{\dagger}(\mathbf{r} \zeta) \frac{\mathbf{p}^{2}}{2 m} \psi(\mathbf{r} \zeta) d^{3} r d \zeta \\
& \hat{W}_{1}=\frac{1}{2} \iint \psi^{\dagger}(\mathbf{r} \zeta) \psi^{\dagger}\left(\mathbf{r}^{\prime} \zeta^{\prime}\right) \frac{e^{2}}{\left|\mathbf{r}-\mathbf{r}^{\prime}\right|} \psi\left(\mathbf{r}^{\prime} \zeta^{\prime}\right) \psi(\mathbf{r} \zeta) d^{3} r d \zeta d^{3} r^{\prime} d \zeta^{\prime} \\
& \hat{W}_{2}=\frac{1}{2} \iint \psi^{\dagger}\left(\mathbf{r}_{1} \zeta_{1}\right) \psi^{\dagger}\left(\mathbf{r}_{2} \zeta_{2}\right) w\left(\mathbf{r}_{1} \zeta_{1} \mathbf{r}_{2} \zeta_{2} ; \mathbf{r}_{3} \zeta_{3} \mathbf{r}_{4} \zeta_{4}\right) \psi\left(\mathbf{r}_{3} \zeta_{3}\right) \psi\left(\mathbf{r}_{4} \zeta_{4}\right) d^{3} r_{1} d \zeta_{1} d^{3} r_{2} d \zeta_{2} d^{3} r_{3} d \zeta_{3} d^{3} r_{4} d \zeta_{4}, \\
& \hat{V}_{1}=\int v_{\text {given }}(\mathbf{r}) \hat{n}(\mathbf{r}) d^{3} r \\
& \hat{V}_{2}=e \int \mathbf{A}_{\text {given }}(\mathbf{r}) \cdot \hat{\mathbf{j}}_{p}^{(T)}(\mathbf{r}) d^{3} r \\
& \hat{V}_{3}=\frac{e^{2}}{2 m} \int \mathbf{A}_{\text {given }}(\mathbf{r})^{2} \hat{n}(\mathbf{r}) d^{3} r
\end{aligned}
$$

where the operators $\hat{V}_{1}, \hat{V}_{2}$ and $\hat{V}_{3}$ denote external potential energies, respectively, and where $\hat{\mathbf{j}}_{p}^{(T)}(\mathbf{r})$ and $\hat{n}(\mathbf{r})$ contained in the external energies are operators of the transverse component of the paramagnetic current-density and the electron density, respectively, which are given by

$$
\begin{aligned}
\hat{\mathbf{j}}_{p}^{(T)}(\mathbf{r}) & =\frac{\hbar}{i 4 \pi m} \int\left[\left\{\nabla_{\mathbf{r}^{\prime}} \psi^{\dagger}\left(\mathbf{r}^{\prime} \zeta^{\prime}\right) \times \nabla_{\mathbf{r}^{\prime}} \psi\left(\mathbf{r}^{\prime} \zeta^{\prime}\right)\right\} \times \frac{\mathbf{r}-\mathbf{r}^{\prime}}{\left|\mathbf{r}-\mathbf{r}^{\prime}\right|^{3}}\right] d^{3} r^{\prime} d \zeta^{\prime}, \\
\hat{n}(\mathbf{r}) & =\int \psi^{\dagger}(\mathbf{r} \zeta) \psi(\mathbf{r} \zeta) d \zeta .
\end{aligned}
$$

Concerning the concrete form of $w\left(\mathbf{r}_{1} \zeta_{1} \mathbf{r}_{2} \zeta_{2} ; \mathbf{r}_{3} \zeta_{3} \mathbf{r}_{4} \zeta_{4}\right)$ in Eq. (8), for example, the phononinduced attractive interaction that gives the BCS reduced Hamiltonian ${ }^{53}-\underline{55}$ is given by

$$
\begin{aligned}
w\left(\mathbf{r}_{1} \zeta_{1} \mathbf{r}_{2} \zeta_{2} ; \mathbf{r}_{3} \zeta_{3} \mathbf{r}_{4} \zeta_{4}\right) & =2 \chi_{\uparrow}\left(\zeta_{1}\right) \chi_{\downarrow}\left(\zeta_{2}\right) \chi_{\downarrow}\left(\zeta_{3}\right) \chi_{\uparrow}\left(\zeta_{4}\right) \\
& \times \sum_{\mathbf{k}} \sum_{\mathbf{k}^{\prime}(\neq \mathbf{k})} V_{\mathbf{k k}^{\prime}} \varphi_{\mathbf{k}^{\prime} \uparrow}\left(\mathbf{r}_{1}\right) \varphi_{-\mathbf{k}^{\prime} \downarrow}\left(\mathbf{r}_{2}\right) \varphi_{-\mathbf{k} \downarrow}^{*}\left(\mathbf{r}_{3}\right) \varphi_{\mathbf{k} \uparrow}^{*}\left(\mathbf{r}_{4}\right),
\end{aligned}
$$

where $V_{\mathbf{k k}^{\prime}}$ is the attractive electron-electron interaction induced by the electron-phonon interaction $\underline{53-55}$, and where $\varphi_{\mathbf{k} \sigma}(\mathbf{r}) \chi_{\sigma}(\zeta)$ is the electron state with the momentum $\mathbf{k}$ and spin $\sigma$.

\section{B. Basic variables}

In the DFT-based or ECS-based theory, we first have to choose the basic variables that determine the properties of the equilibrium state of the system. The OPSS, which is given by Eq. (11), should be chosen as one of basic variables because the superconducting properties 
are reflected in the OPSS as mentioned in Sec. II. Since this is generally a complex number, the complex conjugate $\Delta^{*}\left(\mathbf{r} \zeta, \mathbf{r}^{\prime} \zeta^{\prime}\right)$ is also the basic variable to be chosen. In addition to these

two quantities, the densities $\left\langle\hat{\mathbf{j}}_{p}^{(T)}(\mathbf{r})\right\rangle$ and $\langle\hat{n}(\mathbf{r})\rangle$ should also be chosen as basic variables because these are coupled with the external potentials and will be shown to become basic variables inevitably in the proof of the HK theorem ${ }^{39}$. Here, the meaning of the bracket is the same as that explained below Eq. (11).

Thus, the basic variables of the CDFT for the superconductor are

$$
\begin{aligned}
& n(\mathbf{r})=\left\langle\int \psi^{\dagger}(\mathbf{r} \zeta) \psi(\mathbf{r} \zeta) d \zeta\right\rangle, \\
& \mathbf{j}_{p}^{(T)}(\mathbf{r})=\frac{\hbar}{i 4 \pi m}\left\langle\int\left[\left\{\nabla_{\mathbf{r}^{\prime}} \psi^{\dagger}\left(\mathbf{r}^{\prime} \zeta^{\prime}\right) \times \nabla_{\mathbf{r}^{\prime}} \psi\left(\mathbf{r}^{\prime} \zeta^{\prime}\right)\right\} \times \frac{\mathbf{r}-\mathbf{r}^{\prime}}{\left|\mathbf{r}-\mathbf{r}^{\prime}\right|^{3}}\right] d^{3} r^{\prime} d \zeta^{\prime}\right\rangle, \\
& \Delta\left(\mathbf{r}_{2} \zeta_{2}, \mathbf{r}_{1} \zeta_{1}\right)=\left\langle\psi\left(\mathbf{r}_{1} \zeta_{1}\right) \psi\left(\mathbf{r}_{2} \zeta_{2}\right)\right\rangle, \\
& \Delta\left(\mathbf{r}_{2} \zeta_{2}, \mathbf{r}_{1} \zeta_{1}\right)^{*}=\left\langle\psi\left(\mathbf{r}_{1} \zeta_{1}\right) \psi\left(\mathbf{r}_{2} \zeta_{2}\right)\right\rangle^{*}
\end{aligned}
$$

\section{Universal energy functional}

We define the universal energy functional as

$$
F\left[n, \mathbf{j}_{p}^{(T)}, \Delta, \Delta^{*}\right]=\operatorname{Min}_{\hat{\rho} \rightarrow n, \mathbf{j}_{p}^{(T)}, \Delta, \Delta^{*}} \operatorname{Tr}\left\{\hat{\rho}\left(\hat{T}+\hat{W}_{1}+\hat{W}_{2}\right)+\frac{1}{\beta} \hat{\rho} \ln \hat{\rho}\right\},
$$

where $\beta=1 /\left(k_{B} T\right)$, and where $k_{B}$ and $T$ are Boltzmann factor and temperature of the system, respectively. The RHS of Eq. (19) means that the minimization of the statistical average of energies and entropy operators that are independent of the external potentials is searched by varying the statistical operator $\hat{\rho}$ within the set of $\hat{\rho}$ 's which yield prescribed following densities

$$
\begin{aligned}
& n(\mathbf{r})=\operatorname{Tr}\{\hat{\rho} \hat{n}(\mathbf{r})\}, \\
& \mathbf{j}_{p}^{(T)}(\mathbf{r})=\operatorname{Tr}\left\{\hat{\rho} \hat{\mathbf{j}}_{p}^{(T)}(\mathbf{r})\right\}, \\
& \Delta\left(\mathbf{r} \zeta, \mathbf{r}^{\prime} \zeta^{\prime}\right)=\operatorname{Tr}\left\{\hat{\rho} \hat{\Delta}\left(\mathbf{r} \zeta, \mathbf{r}^{\prime} \zeta^{\prime}\right)\right\} \\
& \Delta^{*}\left(\mathbf{r} \zeta, \mathbf{r}^{\prime} \zeta^{\prime}\right)=\operatorname{Tr}\left\{\hat{\rho} \hat{\Delta}^{\dagger}\left(\mathbf{r} \zeta, \mathbf{r}^{\prime} \zeta^{\prime}\right)\right\}
\end{aligned}
$$

Using this universal energy functional, the HK theorem of the CDFT for the superconductor can be proved, which is shown in the next subsection. 


\section{Hohenberg-Kohn theorem}

The HK theorem consists of two kinds of theorems. One is the variational principle with respect to densities given by Eqs. (201) - (23), and the other is the one-to-one correspondence between the correct statistical operator $\hat{\rho}_{0}$ and the densities of the equilibrium state. These theorems are the fundament of the CDFT for the superconductor. In what follows, we shall give the proof of the HK theorem.

Gibbs's variational principle is written as $\frac{25}{2}$

$$
J_{0}=\operatorname{Min}_{\hat{\rho}} J[\hat{\rho}]=J\left[\hat{\rho}_{0}\right],
$$

with

$$
J[\hat{\rho}]=\operatorname{Tr}\left\{\hat{\rho}(\hat{H}-\mu \hat{N})+\frac{1}{\beta} \hat{\rho} \ln \hat{\rho}\right\},
$$

where $\hat{N}$ is the operator of the electron number, and where $\mu$ is the chemical potential of the system. In Eq. (24), $J_{0}$ is the grand potential of the equilibrium state, and the minimizing statistical operator corresponds to the correct one, which is given by

$$
\hat{\rho}_{0}=\frac{e^{-\beta(\hat{H}-\mu \hat{N})}}{\Xi},
$$

with $\Xi=\operatorname{Tr}\left(e^{-\beta(\hat{H}-\mu \hat{N})}\right)$. This variational principles can be rewritten using two-step variations such that

$$
J_{0}=\operatorname{Min}_{\hat{\rho}} J[\hat{\rho}]=\operatorname{Min}_{n, \mathbf{j}_{p}^{(T)}, \Delta, \Delta^{*}}\left\{\operatorname{Min}_{\hat{\rho} \rightarrow n, \mathbf{j}_{p}^{(T)}, \Delta, \Delta^{*}} J[\hat{\rho}]\right\} .
$$

Substituting Eqs. (25) and (5) into Eq. (27), and using Eqs. (91) - (11) together with Eqs. (20) and (21), we get

$$
\begin{array}{r}
J_{0}=\operatorname{Min}_{n, \mathbf{j}_{p}^{(T)}, \Delta, \Delta^{*}}\left\{F\left[n, \mathbf{j}_{p}^{(T)}, \Delta, \Delta^{*}\right]+\int\left\{v_{\text {given }}(\mathbf{r})-\mu\right\} n(\mathbf{r}) d^{3} r\right. \\
\left.+e \int \mathbf{A}_{\text {given }}(\mathbf{r}) \cdot \mathbf{j}_{p}^{(T)}(\mathbf{r}) d^{3} r+\frac{e^{2}}{2 m} \int \mathbf{A}_{\text {given }}(\mathbf{r})^{2} n(\mathbf{r}) d^{3} r\right\},
\end{array}
$$

where Eq. (19) is used. If we define the following functional

$$
\begin{array}{r}
J_{v_{\text {given }}-\mu, \mathbf{A}_{\text {given }}\left[n, \mathbf{j}_{p}^{(T)}, \Delta, \Delta^{*}\right]}=F\left[n, \mathbf{j}_{p}^{(T)}, \Delta, \Delta^{*}\right]+\int\left\{v_{\text {given }}(\mathbf{r})-\mu\right\} n(\mathbf{r}) d^{3} r \\
+e \int \mathbf{A}_{\text {given }}(\mathbf{r}) \cdot \mathbf{j}_{p}^{(T)}(\mathbf{r}) d^{3} r+\frac{e^{2}}{2 m} \int \mathbf{A}_{\text {given }}(\mathbf{r})^{2} n(\mathbf{r}) d^{3} r
\end{array}
$$


then Eq. (28) is rewritten as

$$
J_{0}=\operatorname{Min}_{n, \mathbf{j}_{p}^{(T)}, \Delta, \Delta^{*}} J^{v_{\text {given }}-\mu, \mathbf{A}_{\text {given }}}\left[n, \mathbf{j}_{p}^{(T)}, \Delta, \Delta^{*}\right]
$$

Compared the second equality of the RHS of Eq. (27) with Eq. (30), the functional $J^{v_{\text {given }}-\mu, \mathbf{A}_{\text {given }}}\left[n, \mathbf{j}_{p}^{(T)}, \Delta, \Delta^{*}\right]$ is written as

$$
J^{v_{\text {given }}-\mu, \mathbf{A}_{\text {given }}}\left[n, \mathbf{j}_{p}^{(T)}, \Delta, \Delta^{*}\right]=\operatorname{Min}_{\hat{\rho} \rightarrow n, \mathbf{j}_{p}^{(T)}, \Delta, \Delta^{*}} J[\hat{\rho}] .
$$

Equation (31) means that the value of $J^{v_{\text {given }}-\mu, \mathbf{A}_{\text {given }}}\left[n, \mathbf{j}_{p}^{(T)}, \Delta, \Delta^{*}\right]$ corresponds to the grand potential at the minimum point within the restricted set of density matrices that yield the prescribed denisties $\left(n, \mathbf{j}_{p}^{(T)}, \Delta, \Delta^{*}\right)$. Therefore, Eq. (30) means that the global minimum point is searched within the set of local minimum points searched by Eq. (31). Since such a global minimum point gives the correct grand potential $J_{0}$, and using Eq. (24), the densities $\left(n, \mathbf{j}_{p}^{(T)}, \Delta, \Delta^{*}\right)$ that are found via Eq. (30) correspond to those that are calculated from the correct statistical operator $\hat{\rho}_{0}$. They are exactly the electron density, transverse component of the paramagnetic current-density and OPSS for the equilibrium state of the system, which are hereafter denoted as $n_{0}, \mathbf{j}_{p 0}^{(T)}, \Delta_{0}$ and $\Delta_{0}^{*}$, respectively. Thus, Eq. (30) represents the variational principle with respect to the electron density, transverse component of the paramagnetic current density and OPSS. The results are summarized as follows:

$$
J_{0}=\underset{n, \mathbf{j}_{p}^{(T)}, \Delta, \Delta^{*}}{\operatorname{Min}} J^{v_{\text {given }}-\mu, \mathbf{A}_{\text {given }}}\left[n, \mathbf{j}_{p}^{(T)}, \Delta, \Delta^{*}\right]=J^{v_{\text {given }}-\mu, \mathbf{A}_{\text {given }}}\left[n_{0}, \mathbf{j}_{p 0}^{(T)}, \Delta_{0}, \Delta_{0}^{*}\right]
$$

with

$$
\begin{aligned}
& n_{0}(\mathbf{r})=\operatorname{Tr}\left\{\hat{\rho}_{0} \hat{n}(\mathbf{r})\right\} \\
& \mathbf{j}_{p 0}^{(T)}(\mathbf{r})=\operatorname{Tr}\left\{\hat{\rho}_{0} \hat{\mathbf{j}}_{p}^{(T)}(\mathbf{r})\right\} \\
& \Delta_{0}\left(\mathbf{r} \zeta, \mathbf{r}^{\prime} \zeta^{\prime}\right)=\operatorname{Tr}\left\{\hat{\rho}_{0} \hat{\Delta}\left(\mathbf{r} \zeta, \mathbf{r}^{\prime} \zeta^{\prime}\right)\right\} \\
& \Delta_{0}^{*}\left(\mathbf{r} \zeta, \mathbf{r}^{\prime} \zeta^{\prime}\right)=\operatorname{Tr}\left\{\hat{\rho}_{0} \hat{\Delta}^{\dagger}\left(\mathbf{r} \zeta, \mathbf{r}^{\prime} \zeta^{\prime}\right)\right\}
\end{aligned}
$$

Next, we shall give the proof of the one-to-one correspondence between the correct statistical operator $\hat{\rho}_{0}$ and equilibrium densities $\left(n_{0}, \mathbf{j}_{p 0}^{(T)}, \Delta_{0}, \Delta_{0}^{*}\right)$. The universal energy func- 
tional at the equilibrium densities is given by

$$
\begin{aligned}
F\left[n_{0}, \mathbf{j}_{p 0}^{(T)}, \Delta_{0}, \Delta_{0}^{*}\right]= & \underset{\hat{\rho} \rightarrow n_{0}, \mathbf{j}_{p 0}^{(T)}, \Delta_{0}, \Delta_{0}^{*}}{\operatorname{Tr}}\left\{\hat{\rho}\left(\hat{T}+\hat{W}_{1}+\hat{W}_{2}\right)+\frac{1}{\beta} \hat{\rho} \ln \hat{\rho}\right\} \\
= & \operatorname{Tr}\left\{\hat{\rho}_{\min }\left[n_{0}, \mathbf{j}_{p 0}^{(T)}, \Delta_{0}, \Delta_{0}^{*}\right]\left(\hat{T}+\hat{W}_{1}+\hat{W}_{2}\right)\right. \\
& \left.+\frac{1}{\beta} \hat{\rho}_{\min }\left[n_{0}, \mathbf{j}_{p 0}^{(T)}, \Delta_{0}, \Delta_{0}^{*}\right] \ln \hat{\rho}_{\min }\left[n_{0}, \mathbf{j}_{p 0}^{(T)}, \Delta_{0}, \Delta_{0}^{*}\right]\right\},
\end{aligned}
$$

where the minimizing statistical operator is referred to as $\hat{\rho}_{\min }\left[n_{0}, \mathbf{j}_{p 0}^{(T)}, \Delta_{0}, \Delta_{0}^{*}\right]$. Taking into account Gibbs's variational principle, i.e., Eq. (24), the following relation holds:

$$
\begin{aligned}
\operatorname{Tr} & \left\{\hat{\rho}_{0}\left(\hat{T}+\hat{W}_{1}+\hat{W}_{2}+\hat{V}_{1}+\hat{V}_{2}+\hat{V}_{3}-\mu \hat{N}\right)+\frac{1}{\beta} \hat{\rho}_{0} \ln \hat{\rho}_{0}\right\} \\
\leq \operatorname{Tr} & \left\{\hat{\rho}_{\min }\left[n_{0}, \mathbf{j}_{p 0}^{(T)}, \Delta_{0}, \Delta_{0}^{*}\right]\left(\hat{T}+\hat{W}_{1}+\hat{W}_{2}+\hat{V}_{1}+\hat{V}_{2}+\hat{V}_{3}-\mu \hat{N}\right)\right. \\
& \left.+\frac{1}{\beta} \hat{\rho}_{\min }\left[n_{0}, \mathbf{j}_{p 0}^{(T)}, \Delta_{0}, \Delta_{0}^{*}\right] \ln \hat{\rho}_{\min }\left[n_{0}, \mathbf{j}_{p 0}^{(T)}, \Delta_{0}, \Delta_{0}^{*}\right]\right\} .
\end{aligned}
$$

Since both $\hat{\rho}_{0}$ and $\hat{\rho}_{\min }\left[n_{0}, \mathbf{j}_{p 0}^{(T)}, \Delta_{0}, \Delta_{0}^{*}\right]$ yield the correct densities $n_{0}, \mathbf{j}_{p 0}^{(T)}, \Delta_{0}, \Delta_{0}^{*}$, Eq. (38) becomes to

$$
\operatorname{Tr}\left\{\hat{\rho}_{0}\left(\hat{T}+\hat{W}_{1}+\hat{W}_{2}\right)+\frac{1}{\beta} \hat{\rho}_{0} \ln \hat{\rho}_{0}\right\} \leq F\left[n_{0}, \mathbf{j}_{p 0}^{(T)}, \Delta_{0}, \Delta_{0}^{*}\right] .
$$

From the definition of $F\left[n_{0}, \mathbf{j}_{p 0}^{(T)}, \Delta_{0}, \Delta_{0}^{*}\right]$, i.e., Eq. (37), only an equal sign is satisfied in Eq. (39). Namely we have

$$
\begin{aligned}
\operatorname{Tr}\left\{\hat{\rho}_{0}\left(\hat{T}+\hat{W}_{1}+\hat{W}_{2}\right)+\frac{1}{\beta} \hat{\rho}_{0} \ln \hat{\rho}_{0}\right\} & =\operatorname{Tr}\left\{\hat{\rho}_{\min }\left[n_{0}, \mathbf{j}_{p 0}^{(T)}, \Delta_{0}, \Delta_{0}^{*}\right]\left(\hat{T}+\hat{W}_{1}+\hat{W}_{2}\right)\right. \\
+ & \left.\frac{1}{\beta} \hat{\rho}_{\min }\left[n_{0}, \mathbf{j}_{p 0}^{(T)}, \Delta_{0}, \Delta_{0}^{*}\right] \ln \hat{\rho}_{\min }\left[n_{0}, \mathbf{j}_{p 0}^{(T)}, \Delta_{0}, \Delta_{0}^{*}\right]\right\}
\end{aligned}
$$

Using the fact that both $\hat{\rho}_{0}$ and $\hat{\rho}_{\min }\left[n_{0}, \mathbf{j}_{p 0}^{(T)}, \Delta_{0}, \Delta_{0}^{*}\right]$ yield the same densities $\left(n_{0}, \mathbf{j}_{p 0}^{(T)}, \Delta_{0}, \Delta_{0}^{*}\right)$, and using Eq. (40), only an equal sign holds also in Eq. (38):

$$
\begin{aligned}
& \operatorname{Tr}\left\{\hat{\rho}_{0}\left(\hat{T}+\hat{W}_{1}+\hat{W}_{2}+\hat{V}_{1}+\hat{V}_{2}+\hat{V}_{3}-\mu \hat{N}\right)+\frac{1}{\beta} \hat{\rho}_{0} \ln \hat{\rho}_{0}\right\} \\
= & \operatorname{Tr}\left\{\hat{\rho}_{\min }\left[n_{0}, \mathbf{j}_{p 0}^{(T)}, \Delta_{0}, \Delta_{0}^{*}\right]\left(\hat{T}+\hat{W}_{1}+\hat{W}_{2}+\hat{V}_{1}+\hat{V}_{2}+\hat{V}_{3}-\mu \hat{N}\right)\right. \\
& \left.+\frac{1}{\beta} \hat{\rho}_{\min }\left[n_{0}, \mathbf{j}_{p 0}^{(T)}, \Delta_{0}, \Delta_{0}^{*}\right] \ln \hat{\rho}_{\min }\left[n_{0}, \mathbf{j}_{p 0}^{(T)}, \Delta_{0}, \Delta_{0}^{*}\right]\right\} .
\end{aligned}
$$

The LHS of Eq. (41) is exactly the correct grand potential $J_{0}$. Considering Gibbs's variational theorem Eq. (24), we finally obtain

$$
\hat{\rho}_{\min }\left[n_{0}, \mathbf{j}_{p 0}^{(T)}, \Delta_{0}, \Delta_{0}^{*}\right]=\hat{\rho}_{0}
$$


It follows that the correct density matrix $\hat{\rho}_{0}\left(=\hat{\rho}_{\min }\left[n_{0}, \mathbf{j}_{p 0}^{(T)}, \Delta_{0}, \Delta_{0}^{*}\right]\right)$ is uniquely determined by the correct densities $\left(n_{0}, \mathbf{j}_{p 0}^{(T)}, \Delta_{0}, \Delta_{0}^{*}\right)$ via Eq. (37), and vice versa due to Eqs. (33) - (36). Thus, it is successfully proved that the one-to-one correspondence between the statistical operator $\hat{\rho}_{0}$ and densities $\left(n_{0}, \mathbf{j}_{p 0}^{(T)}, \Delta_{0}, \Delta_{0}^{*}\right)$ holds.

\section{SINGLE-PARTICLE EQUATION IN THE REFERENCE SYSTEM}

In order to reproduce the equilibrium densities $\left(n_{0}, \mathbf{j}_{p 0}^{(T)}, \Delta_{0}, \Delta_{0}^{*}\right)$, we shall introduce the reference system in a similar way to the ECS theory $\underline{\underline{39}} \underline{\underline{40}}$. The reference system is the noninteracting system in which there exist the mean-field potentials applied so as to make basic variables coincide with the equilibrium densities.

Since it is in the quadratic form in terms of the field operator of electrons, the Hamiltonian of the reference system can be diagonalized in terms of the noninteracting fermion quasiparticle via the so-called Bogoliubov-Valatin (BV) transformation $\underline{56}, 57$. The matrix elements of the BV transformation can be obtained by solving the single-particle equation which is called the BdG-KS equation. In this section we also derive the BdG-KS equation and confirm its solutions to be satisfied with orthonormal and complete properties caused from the property of the BV transformation.

\section{A. Reference system}

We introduce the noninteracting system as the reference system in which the effective mean-field potentials are applied instead of the interaction energy term consisting of four field operators of electrons. The Hamiltonian of the reference system is given by

$$
\begin{aligned}
\hat{H}_{s}= & \int \psi^{\dagger}(\mathbf{r} \zeta)\left[\frac{1}{2 m}\left\{\mathbf{p}+e \mathbf{A}_{s}(\mathbf{r})\right\}^{2}\right] \psi(\mathbf{r} \zeta) d^{3} r d \zeta \\
& +\int v_{s}(\mathbf{r}) \psi^{\dagger}(\mathbf{r} \zeta) \psi(\mathbf{r} \zeta) d^{3} r d \zeta \\
& +\iint D_{s}^{*}\left(\mathbf{r} \zeta, \mathbf{r}^{\prime} \zeta^{\prime}\right) \hat{\Delta}\left(\mathbf{r} \zeta, \mathbf{r}^{\prime} \zeta^{\prime}\right) d^{3} r d \zeta d^{3} r^{\prime} d \zeta^{\prime} \\
& +\iint D_{s}\left(\mathbf{r} \zeta, \mathbf{r}^{\prime} \zeta^{\prime}\right) \hat{\Delta}^{\dagger}\left(\mathbf{r} \zeta, \mathbf{r}^{\prime} \zeta^{\prime}\right) d^{3} r d \zeta d^{3} r^{\prime} d \zeta^{\prime}
\end{aligned}
$$

where $v_{s}(\mathbf{r}), \mathbf{A}_{s}(\mathbf{r}), D_{s}\left(\mathbf{r} \zeta, \mathbf{r}^{\prime} \zeta^{\prime}\right)$ and $D_{s}^{*}\left(\mathbf{r} \zeta, \mathbf{r}^{\prime} \zeta^{\prime}\right)$ are effective mean-field potentials. Especially $D_{s}\left(\mathbf{r} \zeta, \mathbf{r}^{\prime} \zeta^{\prime}\right)$ is called the effective pair potential which induces the OPSS to be nonzero 
when the system is in the superconducting state. The concrete forms of these effective potentials are determined by using the HK theorem, which will be shown in Sec. V.

The essential point about the reference system is that the Hamiltonian (43) includes the pair potential terms, i.e., the 3rd and 4th terms, which explicitly breaks the conservation of the electron number. In other words, we intentionally prepare for the reference system which can express the symmetry breaking state of the $U(\mathbb{I})$ gauge. This device for the reference system is similar to that for the mean-field approximation of the BCS theory $\underline{53}$.

Let us consider the transformation from the system of electrons to that of the fermion quasiparticles, annihilation and creation operators of which are denoted as $\gamma_{i}$ and $\gamma_{i}^{\dagger}$, respectively. Since it is quadratic in terms of the field operator of electrons, the Hamiltonian (43) can be diagonalized in terms of the fermion quasiparticle via the BV transformation $\frac{56,57}{5}$. Suppose that the transformation is given by

$$
\begin{aligned}
& \psi(\mathbf{r} \zeta)=\sum_{i} u_{i}(\mathbf{r} \zeta) \gamma_{i}+\sum_{j} v_{j}(\mathbf{r} \zeta) \gamma_{j}^{\dagger}, \\
& \psi^{\dagger}(\mathbf{r} \zeta)=\sum_{i} u_{i}^{*}(\mathbf{r} \zeta) \gamma_{i}^{\dagger}+\sum_{j} v_{j}^{*}(\mathbf{r} \zeta) \gamma_{j},
\end{aligned}
$$

where $u_{i}(\mathbf{r} \zeta)$ and $v_{i}(\mathbf{r} \zeta)$ correspond to the matrix elements of the BV transformation. They are determined by requiring $\hat{H}_{s}$ to be diagonalized in terms of $\gamma_{i}$ and $\gamma_{i}^{\dagger}$.

Substituting Eq. (44) into the aniticommutation relations for $\psi(\mathbf{r} \zeta)$ and $\psi^{\dagger}(\mathbf{r} \zeta)$, and using the anticommutation relations for $\gamma_{i}$ and $\gamma_{i}^{\dagger}$, the following relations hold:

$$
\begin{aligned}
& \sum_{i}\left\{u_{i}^{*}(\mathbf{r} \zeta) u_{i}\left(\mathbf{r}^{\prime} \zeta^{\prime}\right)+v_{i}^{*}(\mathbf{r} \zeta) v_{i}\left(\mathbf{r}^{\prime} \zeta^{\prime}\right)\right\}=\delta\left(\mathbf{r}-\mathbf{r}^{\prime}\right) \delta_{\zeta \zeta^{\prime}} \\
& \sum_{i}\left\{u_{i}(\mathbf{r} \zeta) v_{i}\left(\mathbf{r}^{\prime} \zeta^{\prime}\right)+v_{i}(\mathbf{r} \zeta) u_{i}\left(\mathbf{r}^{\prime} \zeta^{\prime}\right)\right\}=0 .
\end{aligned}
$$

In other words, Eqs. (45) and (46) have to be satisfied so that Eq. (44) is consistent with both anticommutation relations for $\psi(\mathbf{r} \zeta)$ and $\psi^{\dagger}(\mathbf{r} \zeta)$ and those for $\gamma_{i}$ and $\gamma_{i}^{\dagger}$. For the purpose of reference, the property of the BV transformation Eq. (44) is given in the Appendix.

\section{B. BdG-KS equation}

In this subsection, we derive the conditions on $u_{i}(\mathbf{r} \zeta)$ and $v_{i}(\mathbf{r} \zeta)$ for diagonalyzing the Hamiltonian $\hat{H}_{s}$ in terms of $\gamma_{i}$ and $\gamma_{i}^{\dagger}$. Suppose that the diagonalized Hamiltonian is given 
by

$$
\hat{H}_{s}-\mu \hat{N}=E_{g}+\sum_{i} E_{i} \gamma_{i}^{\dagger} \gamma_{i},
$$

where $E_{g}$ and $E_{i}$ are the ground-state energy and excited-state energies, respectively. Using Eq. (47) and anticommutation relations for $\gamma_{i}$ and $\gamma_{i}^{\dagger}$, we have

$$
\begin{aligned}
& {\left[\gamma_{i}, \hat{H}_{s}-\mu \hat{N}\right]=E_{i} \gamma_{i},} \\
& {\left[\gamma_{i}^{\dagger}, \hat{H}_{s}-\mu \hat{N}\right]=-E_{i} \gamma_{i}^{\dagger} .}
\end{aligned}
$$

Also, using Eq. (43) and aniticommutation relations for $\psi(\mathbf{r} \zeta)$ and $\psi^{\dagger}(\mathbf{r} \zeta)$, we have

$$
\begin{aligned}
{\left[\psi(\mathbf{r} \zeta), \hat{H}_{s}-\mu \hat{N}\right]=} & \left(h_{s}^{\mathbf{r}}-\mu\right) \psi(\mathbf{r} \zeta) \\
& +\int\left\{D_{s}\left(\mathbf{r} \zeta, \mathbf{r}^{\prime} \zeta^{\prime}\right)-D_{s}\left(\mathbf{r}^{\prime} \zeta^{\prime}, \mathbf{r} \zeta\right)\right\} \psi^{\dagger}\left(\mathbf{r}^{\prime} \zeta^{\prime}\right) d^{3} r^{\prime} d \zeta^{\prime}, \\
{\left[\psi^{\dagger}(\mathbf{r} \zeta), \hat{H}_{s}-\mu \hat{N}\right]=} & -\int \psi^{\dagger}\left(\mathbf{r}^{\prime} \zeta^{\prime}\right)\left(h_{s}^{\mathbf{r}^{\prime}}-\mu\right) \delta\left(\mathbf{r}-\mathbf{r}^{\prime}\right) \delta_{\zeta \zeta^{\prime}} d^{3} r^{\prime} d \zeta^{\prime} \\
& +\int\left\{D_{s}^{*}\left(\mathbf{r}^{\prime} \zeta^{\prime}, \mathbf{r} \zeta\right)-D_{s}^{*}\left(\mathbf{r} \zeta, \mathbf{r}^{\prime} \zeta^{\prime}\right)\right\} \psi\left(\mathbf{r}^{\prime} \zeta^{\prime}\right) d^{3} r^{\prime} d \zeta^{\prime},
\end{aligned}
$$

where $h_{s}^{\mathrm{r}}$ is defined as

$$
h_{s}^{\mathbf{r}}=\frac{1}{2 m}\left\{\mathbf{p}+e \mathbf{A}_{s}(\mathbf{r})\right\}^{2}+v_{s}(\mathbf{r}) .
$$

Substituting Eq. (44) into Eq. (50), and using Eqs. (48) and (49), we finally obtain the single-particle equation with which $u_{i}(\mathbf{r} \zeta)$ and $v_{i}(\mathbf{r} \zeta)$ should be satisfied:

$$
\begin{aligned}
& \left(h_{s}^{\mathbf{r}}-\mu\right) u_{i}(\mathbf{r} \zeta)+\int \tilde{D}_{s}\left(\mathbf{r} \zeta, \mathbf{r}^{\prime} \zeta^{\prime}\right) v_{i}^{*}\left(\mathbf{r}^{\prime} \zeta^{\prime}\right) d^{3} r^{\prime} d \zeta^{\prime}=E_{i} u_{i}(\mathbf{r} \zeta), \\
& -\left(h_{s}^{\mathbf{r}}-\mu\right) v_{i}(\mathbf{r} \zeta)-\int \tilde{D}_{s}\left(\mathbf{r} \zeta, \mathbf{r}^{\prime} \zeta^{\prime}\right) u_{i}^{*}\left(\mathbf{r}^{\prime} \zeta^{\prime}\right) d^{3} r^{\prime} d \zeta^{\prime}=E_{i} v_{i}(\mathbf{r} \zeta),
\end{aligned}
$$

where

$$
\tilde{D}_{s}\left(\mathbf{r} \zeta, \mathbf{r}^{\prime} \zeta^{\prime}\right)=D_{s}\left(\mathbf{r} \zeta, \mathbf{r}^{\prime} \zeta^{\prime}\right)-D_{s}\left(\mathbf{r}^{\prime} \zeta^{\prime}, \mathbf{r} \zeta\right) .
$$

The solutions of Eq. (53) correspond to the matrix elements of the BV transformation which makes the Hamiltonian Eq. (43) be diagonalized in the form of Eq. (47). The equation (53) is called the BdG-KS equation $\underline{44} \underline{\underline{48}}$. As shown in the next subsection, the eigenvalue $E_{i}$ is a real number. Using this result, it is shown that Eq. (53) is also obtained from starting with Eq. (51) instead of Eq. (50). 


\section{Orthonormality for the solution of the BdG-KS equation}

In this subsection, we discuss the orthonormal properties of the solution of the BdGKS equation that are used in developing the approximate form of the xc energy functional (Sec. VI). First we show that the eigenvalue $E_{i}$ of Eq. (53) is a real number. Multiplying both sides of the first (second) equation of Eq. (53) by $u_{i}^{*}(\mathbf{r} \zeta)\left(v_{i}^{*}(\mathbf{r} \zeta)\right)$ from the left, and integrating with respect to $\mathbf{r}$ and $\zeta$, yield

$$
\begin{aligned}
& \int u_{i}^{*}(\mathbf{r} \zeta)\left(h_{s}^{\mathbf{r}}-\mu\right) u_{i}(\mathbf{r} \zeta) d^{3} r d \zeta \\
& +\iint u_{i}^{*}(\mathbf{r} \zeta) \tilde{D}_{s}\left(\mathbf{r} \zeta, \mathbf{r}^{\prime} \zeta^{\prime}\right) v_{i}^{*}\left(\mathbf{r}^{\prime} \zeta^{\prime}\right) d^{3} r^{\prime} d \zeta^{\prime} d^{3} r d \zeta=E_{i} \int\left|u_{i}(\mathbf{r} \zeta)\right|^{2} d^{3} r d \zeta \\
& -\int v_{i}^{*}(\mathbf{r} \zeta)\left(h_{s}^{\mathbf{r}}-\mu\right) v_{i}(\mathbf{r} \zeta) d^{3} r d \zeta \\
& +\iint u_{i}^{*}(\mathbf{r} \zeta) \tilde{D}_{s}\left(\mathbf{r} \zeta, \mathbf{r}^{\prime} \zeta^{\prime}\right) v_{i}^{*}\left(\mathbf{r}^{\prime} \zeta^{\prime}\right) d^{3} r^{\prime} d \zeta^{\prime} d^{3} r d \zeta=E_{i} \int\left|v_{i}(\mathbf{r} \zeta)\right|^{2} d^{3} r d \zeta
\end{aligned}
$$

respectively. Subtracting Eq. (56) from Eq. (551) on both sides, we have

$$
\begin{aligned}
& \int u_{i}^{*}(\mathbf{r} \zeta)\left(h_{s}^{\mathbf{r}}-\mu\right) u_{i}(\mathbf{r} \zeta) d^{3} r d \zeta+\int v_{i}^{*}(\mathbf{r} \zeta)\left(h_{s}^{\mathbf{r}}-\mu\right) v_{i}(\mathbf{r} \zeta) d^{3} r d \zeta \\
= & E_{i} \int\left\{\left|u_{i}(\mathbf{r} \zeta)\right|^{2}-\left|v_{i}(\mathbf{r} \zeta)\right|^{2}\right\} d^{3} r d \zeta .
\end{aligned}
$$

Since both terms of the LHS are real numbers due to the Hermitian property of Eq. (52) and since the integration of the RHS is a real number, $E_{i}$ is necessarily a real number. Using this result, Eq. (53) is rewritten as

$$
\hat{\Lambda}\left(\begin{array}{c}
u_{i}(\mathbf{r} \zeta) \\
v_{i}^{*}(\mathbf{r} \zeta)
\end{array}\right)=E_{i}\left(\begin{array}{c}
u_{i}(\mathbf{r} \zeta) \\
v_{i}^{*}(\mathbf{r} \zeta)
\end{array}\right),
$$

where $\hat{\Lambda}$ is a $2 \times 2$ matrix defined as

$$
\hat{\Lambda}=\left(\begin{array}{cc}
h_{s}^{\mathbf{r}}-\mu & \hat{D}_{s} \\
-\hat{D}_{s}^{*} & -\left(h_{s}^{\mathbf{r}}-\mu\right)^{*}
\end{array}\right),
$$

and where $\hat{D}_{s}$ is defined as the operator which acts the function $f(\mathbf{r} \zeta)$ such that

$$
\hat{D}_{s} f(\mathbf{r} \zeta)=\int \tilde{D}_{s}\left(\mathbf{r} \zeta, \mathbf{r}^{\prime} \zeta^{\prime}\right) f\left(\mathbf{r}^{\prime} \zeta^{\prime}\right) d^{3} r^{\prime} d \zeta^{\prime}
$$


The solution $\left(\begin{array}{c}u_{i}(\mathbf{r} \zeta) \\ v_{i}^{*}(\mathbf{r} \zeta)\end{array}\right)$ can be regarded as the eigenstate of Eq. (158). It is easily shown that the matrix elements

$$
\Lambda_{i j}=\int\left(u_{i}(\mathbf{r} \zeta) v_{i}^{*}(\mathbf{r} \zeta)\right)^{*} \hat{\Lambda}\left(\begin{array}{c}
u_{j}(\mathbf{r} \zeta) \\
v_{j}^{*}(\mathbf{r} \zeta)
\end{array}\right) d^{3} r d \zeta
$$

are satisfied with the Hermitian property $\Lambda_{i j}=\Lambda_{j i}^{*}$. Using this property, it is also shown in a usual way $\underline{58}$ that the eigenstates of Eq. (158) can be chosen to have the orthonormal property

$$
\int\left\{u_{i}^{*}(\mathbf{r} \zeta) u_{j}(\mathbf{r} \zeta)+v_{i}(\mathbf{r} \zeta) v_{j}^{*}(\mathbf{r} \zeta)\right\} d^{3} r d \zeta=\delta_{i j}
$$

Further, the other type of orthonormal property is obtained by utilizing the solution of the BdG-KS equation. It is confirmed that the BdG-KS equation (58) can be rewritten as

$$
\hat{\Lambda}\left(\begin{array}{c}
v_{i}(\mathbf{r} \zeta) \\
u_{i}^{*}(\mathbf{r} \zeta)
\end{array}\right)=-E_{i}\left(\begin{array}{c}
v_{i}(\mathbf{r} \zeta) \\
u_{i}^{*}(\mathbf{r} \zeta)
\end{array}\right)
$$

Namely, $\left(\begin{array}{c}v_{i}(\mathbf{r} \zeta) \\ u_{i}^{*}(\mathbf{r} \zeta)\end{array}\right)$ is also the solution of the BdG-KS equation, and the corresponding eigenvalue is $-E_{i}$. Since the excitation energy of the quasiparticle is supposed to be positive, i.e., $E_{i} \neq-E_{j}$, we have

$$
\int\left\{u_{i}^{*}(\mathbf{r} \zeta) v_{j}(\mathbf{r} \zeta)+v_{i}(\mathbf{r} \zeta) u_{j}^{*}(\mathbf{r} \zeta)\right\} d^{3} r d \zeta=0
$$

In the special case $i=j$, Eq. (64) becomes to

$$
\int u_{i}^{*}(\mathbf{r} \zeta) v_{i}(\mathbf{r} \zeta) d^{3} r d \zeta=0
$$

Equations (45) and (46) can be regarded as a kind of completeness of the solutions of Eq. (58) because such relations contain the summation on the number $i$ specifying the eigenstates of Eq. (58). On the other hand, Eqs. (62), (64) and (65) can be regarded as the orthonormal property of the solutions of Eq. (58).

\section{EFFECTIVE MEAN-FIELD POTENTIALS}

In this section we present the explicit forms of the effective mean-field potentials $v_{s}(\mathbf{r})$, $\mathbf{A}_{s}(\mathbf{r}), D_{s}\left(\mathbf{r} \zeta, \mathbf{r}^{\prime} \zeta^{\prime}\right)$ and $D_{s}^{*}\left(\mathbf{r} \zeta, \mathbf{r}^{\prime} \zeta^{\prime}\right)$. They are determined by requiring the basic variables calculated in the reference system to be in accordance with the equilibrium densities. 


\section{A. The HK theorem for the reference system}

First, we consider the HK theorem for the reference system. Since the proof is similar to that of the real system (Sec. III), we shall show only the outline of this theorem. The Hamiltonian of the reference system is given by Eq. (43). In a similar way to Eq. (5), neglecting the surface integral at the infinite distance and adopting the Coulomb gauge given by $\nabla \cdot \mathbf{A}_{s}(\mathbf{r})=0$, Eq. (43) is rewritten as

$$
\hat{H}_{s}=\hat{T}+\hat{V}_{1 s}+\hat{V}_{2 s}+\hat{V}_{3 s}+\hat{V}_{D s}
$$

where $\hat{T}$ is given by Eq. (6), and where

$$
\begin{aligned}
\hat{V}_{1 s}= & \int v_{s}(\mathbf{r}) \hat{n}(\mathbf{r}) d^{3} r \\
\hat{V}_{2 s}= & e \int \mathbf{A}_{s}(\mathbf{r}) \cdot \hat{\mathbf{j}}_{p}^{(T)}(\mathbf{r}) d^{3} r \\
\hat{V}_{3 s}= & \frac{e^{2}}{2 m} \int \mathbf{A}_{s}(\mathbf{r})^{2} \hat{n}(\mathbf{r}) d^{3} r \\
\hat{V}_{D s}= & \iint D_{s}^{*}\left(\mathbf{r} \zeta, \mathbf{r}^{\prime} \zeta^{\prime}\right) \hat{\Delta}\left(\mathbf{r} \zeta, \mathbf{r}^{\prime} \zeta^{\prime}\right) d^{3} r d \zeta d^{3} r^{\prime} d \zeta^{\prime} \\
& +\iint D_{s}\left(\mathbf{r} \zeta, \mathbf{r}^{\prime} \zeta^{\prime}\right) \hat{\Delta}^{*}\left(\mathbf{r} \zeta, \mathbf{r}^{\prime} \zeta^{\prime}\right) d^{3} r d \zeta d^{3} r^{\prime} d \zeta^{\prime}
\end{aligned}
$$

The universal energy functional for the reference system is defined as

$$
F_{s}\left[n, \mathbf{j}_{p}^{(T)}, \Delta, \Delta^{*}\right]=\operatorname{Min}_{\hat{\rho} \rightarrow n, \mathbf{j}_{p}^{(T)}, \Delta, \Delta^{*}} \operatorname{Tr}\left\{\hat{\rho} \hat{T}+\frac{1}{\beta} \hat{\rho} \ln \hat{\rho}\right\} .
$$

Gibbs's variational principle holds also for the reference system. If we define the following functional

$$
J_{s}[\hat{\rho}]=\operatorname{Tr}\left\{\hat{\rho}\left(\hat{H}_{s}-\mu \hat{N}\right)+\frac{1}{\beta} \hat{\rho} \ln \hat{\rho}\right\},
$$

then the minimum point $J_{s 0}$ exists at the statistical operator $\hat{\rho}_{s 0}$ :

$$
J_{s 0}=\operatorname{Min}_{\hat{\rho}} J_{s}[\hat{\rho}]=J_{s}\left[\hat{\rho}_{s 0}\right]
$$

where

$$
\hat{\rho}_{s 0}=\frac{e^{-\beta\left(\hat{H}_{s}-\mu \hat{N}\right)}}{\Xi_{s}}
$$

with $\Xi_{s}=\operatorname{Tr}\left(e^{-\beta\left(\hat{H}_{s}-\mu \hat{N}\right)}\right)$. In a similar way to the case of the real system, the variational principles Eq. (73) can be rewritten as the variational principle with respect to the densities 
chosen as the basic variables, i.e., $\left(n, \mathbf{j}_{p}^{(T)}, \Delta, \Delta^{*}\right)$. We have

$$
J_{s 0}=\operatorname{Min}_{n, \mathbf{j}_{p}^{(T)}, \Delta, \Delta^{*}} J_{s}^{v_{s}-\mu, \mathbf{A}_{s}, D_{s}, D_{s}^{*}}\left[n, \mathbf{j}_{p}^{(T)}, \Delta, \Delta^{*}\right]
$$

where

$$
\begin{aligned}
& J_{s}^{v_{s}-\mu, \mathbf{A}_{s}, D_{s}, D_{s}^{*}}\left[n, \mathbf{j}_{p}^{(T)}, \Delta, \Delta^{*}\right]=F_{s}\left[n, \mathbf{j}_{p}^{(T)}, \Delta, \Delta^{*}\right]+\int\left\{v_{s}(\mathbf{r})-\mu\right\} n(\mathbf{r}) d^{3} r \\
& +e \int \mathbf{A}_{s}(\mathbf{r}) \cdot \mathbf{j}_{p}^{(T)}(\mathbf{r}) d^{3} r+\frac{e^{2}}{2 m} \int \mathbf{A}_{s}(\mathbf{r})^{2} n(\mathbf{r}) d^{3} r \\
& +\iint D_{s}^{*}\left(\mathbf{r} \zeta, \mathbf{r}^{\prime} \zeta^{\prime}\right) \Delta\left(\mathbf{r} \zeta, \mathbf{r}^{\prime} \zeta^{\prime}\right) d^{3} r d \zeta d^{3} r^{\prime} d \zeta^{\prime} \\
& +\iint D_{s}\left(\mathbf{r} \zeta, \mathbf{r}^{\prime} \zeta^{\prime}\right) \Delta^{*}\left(\mathbf{r} \zeta, \mathbf{r}^{\prime} \zeta^{\prime}\right) d^{3} r d \zeta d^{3} r^{\prime} d \zeta^{\prime}
\end{aligned}
$$

The minimizing densities searched in Eq. (75), which are denoted as $n_{s 0}, \mathbf{j}_{p s 0}^{(T)}, \Delta_{s 0}$ and $\Delta_{s 0}^{*}$, correspond to those calculated by means of Eq. (174). That is,

$$
J_{s 0}=J_{s}^{v_{s}-\mu, \mathbf{A}_{s}, D_{s}, D_{s}^{*}}\left[n_{s 0}, \mathbf{j}_{p s 0}^{(T)}, \Delta_{s 0}, \Delta_{s 0}^{*}\right]
$$

with

$$
\begin{aligned}
& n_{s 0}(\mathbf{r})=\operatorname{Tr}\left\{\hat{\rho}_{s 0} \hat{n}(\mathbf{r})\right\} \\
& \mathbf{j}_{p s 0}^{(T)}(\mathbf{r})=\operatorname{Tr}\left\{\hat{\rho}_{s 0} \hat{\mathbf{j}}_{p}^{(T)}(\mathbf{r})\right\} \\
& \Delta_{s 0}\left(\mathbf{r} \zeta, \mathbf{r}^{\prime} \zeta^{\prime}\right)=\operatorname{Tr}\left\{\hat{\rho}_{s 0} \hat{\Delta}\left(\mathbf{r} \zeta, \mathbf{r}^{\prime} \zeta^{\prime}\right)\right\} \\
& \Delta_{s 0}^{*}\left(\mathbf{r} \zeta, \mathbf{r}^{\prime} \zeta^{\prime}\right)=\operatorname{Tr}\left\{\hat{\rho}_{s 0} \hat{\Delta}^{\dagger}\left(\mathbf{r} \zeta, \mathbf{r}^{\prime} \zeta^{\prime}\right)\right\}
\end{aligned}
$$

This is the variational principle with respect to the densities in the reference system. As mentioned later, the effective mean-field potentials are determined so that $\left(n_{s 0}, \mathbf{j}_{p s 0}^{(T)}, \Delta_{s 0}, \Delta_{s 0}^{*}\right)$ coincide with the equilibrium densities of the real system, i.e., $\left(n_{0}, \mathbf{j}_{p 0}^{(T)}, \Delta_{0}, \Delta_{0}^{*}\right)$ given by Eqs. (33) - (36).

The one-to-one correspondence between the statistical operator $\hat{\rho}_{s 0}$ and densities $\left(n_{s 0}, \mathbf{j}_{p s 0}^{(T)}, \Delta_{s 0}, \Delta_{s 0}^{*}\right)$ can be proven similarly to the case of the real system (Sec. III). Namely, if the minimizing statistical operator of Eq. (171) that yields the prescribed densities $\left(n_{s 0}, \mathbf{j}_{p s 0}^{(T)}, \Delta_{s 0}, \Delta_{s 0}^{*}\right)$ is denoted as $\hat{\rho}_{s, \min }\left[n_{s 0}, \mathbf{j}_{p s 0}^{(T)}, \Delta_{s 0}, \Delta_{s 0}^{*}\right]$, then we can obtain

$$
\hat{\rho}_{s, \min }\left[n_{s 0}, \mathbf{j}_{p s 0}^{(T)}, \Delta_{s 0}, \Delta_{s 0}^{*}\right]=\hat{\rho}_{s 0} .
$$

Thus, the one-to-one correspondence between $\hat{\rho}_{s 0}$ and densities $\left(n_{s 0}, \mathbf{j}_{p s 0}^{(T)}, \Delta_{s 0}, \Delta_{s 0}^{*}\right)$ holds in the reference system. 


\section{B. Effective mean-field potentials}

According to the HK theorem for the reference system, the functional $J_{s}^{v_{s}-\mu, \mathbf{A}_{s}, D_{s}, D_{s}^{*}}\left[n, \mathbf{j}_{p}^{(T)}, \Delta, \Delta^{*}\right]$ takes the minimum value at densities $\left(n_{s 0}, \mathbf{j}_{p s 0}^{(T)}, \Delta_{s 0}, \Delta_{s 0}^{*}\right)$. Namely, substituting Eq. (76) into

$$
\begin{aligned}
& J_{s}^{v_{s}-\mu, \mathbf{A}_{s}, D_{s}, D_{s}^{*}}\left[n_{s 0}+\delta n, \mathbf{j}_{p s 0}^{(T)}+\delta \mathbf{j}_{p}^{(T)}, \Delta_{s 0}+\delta \Delta, \Delta_{s 0}^{*}+\delta \Delta^{*}\right] \\
& -J_{s}^{v_{s}-\mu, \mathbf{A}_{s}, D_{s}, D_{s}^{*}}\left[n_{s 0}, \mathbf{j}_{p s 0}^{(T)}, \Delta_{s 0}, \Delta_{s 0}^{*}\right]=0,
\end{aligned}
$$

leads to

$$
\begin{aligned}
& \left.\frac{\delta F_{s}\left[n, \mathbf{j}_{p}^{(T)}, \Delta, \Delta^{*}\right]}{\delta n(\mathbf{r})}\right|_{\substack{n=n_{s 0} \\
\mathbf{j}_{p}^{(T)}=\mathbf{j}_{p s 0}^{(T)} \\
\Delta=\Delta_{s 0} \\
\Delta^{*}=\Delta_{s 0}^{*}}}+\left\{v_{s}(\mathbf{r})-\mu\right\}+\frac{e^{2}}{2 m} \mathbf{A}_{s}(\mathbf{r})^{2}=0, \\
& \left.\frac{\delta F_{s}\left[n, \mathbf{j}_{p}^{(T)}, \Delta, \Delta^{*}\right]}{\delta \mathbf{j}_{p}^{(T)}(\mathbf{r})}\right|_{\substack{n=n_{s 0} \\
\mathbf{j}_{p}^{(T)}=\mathbf{j}_{p s 0}^{(T)}}}+e \mathbf{A}_{s}(\mathbf{r})=0, \\
& \Delta=\Delta_{s 0} \\
& \Delta^{*}=\Delta_{s 0}^{*} \\
& \left.\frac{\delta F_{s}\left[n, \mathbf{j}_{p}^{(T)}, \Delta, \Delta^{*}\right]}{\delta \Delta\left(\mathbf{r} \zeta, \mathbf{r}^{\prime} \zeta^{\prime}\right)}\right|_{\substack{n=n_{s 0} \\
\mathbf{j}_{p}^{(T)} \mathbf{j}_{p s 0}^{(T)} \\
\Delta=\Delta_{s 0} \\
\Delta^{*}=\Delta_{s 0}^{*}}}+D_{s}^{*}\left(\mathbf{r} \zeta, \mathbf{r}^{\prime} \zeta^{\prime}\right)=0, \\
& \left.\frac{\delta F_{s}\left[n, \mathbf{j}_{p}^{(T)}, \Delta, \Delta^{*}\right]}{\delta \Delta^{*}\left(\mathbf{r} \zeta, \mathbf{r}^{\prime} \zeta^{\prime}\right)}\right|_{\substack{n=n_{s 0} \\
\mathbf{j}_{p}^{(T)}=\mathbf{j}_{p s 0}^{(T)}}}+D_{s}\left(\mathbf{r} \zeta, \mathbf{r}^{\prime} \zeta^{\prime}\right)=0 . \\
& \Delta=\Delta_{s 0} \\
& \Delta^{*}=\Delta_{s 0}^{*}
\end{aligned}
$$

Using $F_{s}\left[n, \mathbf{j}_{p}^{(T)}, \Delta, \Delta^{*}\right]$ and the classical Coulomb interactions between electrons, the universal energy functional of the real system, i.e., $F\left[n, \mathbf{j}_{p}^{(T)}, \Delta, \Delta^{*}\right]$, is formally decomposed into the following form:

$$
F\left[n, \mathbf{j}_{p}^{(T)}, \Delta, \Delta^{*}\right]=F_{s}\left[n, \mathbf{j}_{p}^{(T)}, \Delta, \Delta^{*}\right]+\frac{e^{2}}{2} \iint \frac{n(\mathbf{r}) n\left(\mathbf{r}^{\prime}\right)}{\left|\mathbf{r}-\mathbf{r}^{\prime}\right|} d^{3} r d^{3} r^{\prime}+F_{x c}\left[n, \mathbf{j}_{p}^{(T)}, \Delta, \Delta^{*}\right],
$$


where $F_{x c}\left[n, \mathbf{j}_{p}^{(T)}, \Delta, \Delta^{*}\right]$ is the xc energy functional which contains the quantum effects of the electron-electron interaction, the difference of the kinetic energy between the real and reference systems, and the difference of the entropy between these two systems. Substituting Eq. (88) into Eq. (29), and using the HK theorem given by Eq. (32), then we have

$$
\begin{aligned}
& \left.\frac{\delta F_{s}\left[n, \mathbf{j}_{p}^{(T)}, \Delta, \Delta^{*}\right]}{\delta n(\mathbf{r})}\right|_{\substack{n=n_{0} \\
\mathbf{j}_{p}^{(T)}=\mathbf{j}_{p 0}^{(T)} \\
\Delta=\Delta_{0} \\
\Delta^{*}=\Delta_{0}^{*}}}+e^{2} \int \frac{n_{0}\left(\mathbf{r}^{\prime}\right)}{\left|\mathbf{r}-\mathbf{r}^{\prime}\right|} d^{3} r^{\prime} \\
& +\left.\frac{\delta F_{x c}\left[n, \mathbf{j}_{p}^{(T)}, \Delta, \Delta^{*}\right]}{\delta n(\mathbf{r})}\right|_{\substack{n=n_{0} \\
\mathbf{j}_{p}^{(T)}=\mathbf{j}_{p 0}^{(T)} \\
\Delta=\Delta_{0} \\
\Delta^{*}=\Delta_{0}^{*}}}+\left\{v_{\text {given }}(\mathbf{r})-\mu\right\}+\frac{e^{2}}{2 m} \mathbf{A}_{\text {given }}(\mathbf{r})^{2}=0, \\
& \frac{\delta F_{s}\left[n, \mathbf{j}_{p}^{(T)}, \Delta, \Delta^{*}\right]}{\delta \mathbf{j}_{p}^{(T)}(\mathbf{r})} \mid \begin{array}{l}
\substack{n=n_{0} \\
\mathbf{j}_{p}^{(T)}=\mathbf{j}_{p 0}^{(T)} \\
\Delta=\Delta_{0} \\
\Delta^{*}=\Delta_{0}^{*}} \\
\substack{n=n_{0} \\
\mathbf{j}_{p}^{(T)}=\mathbf{j}_{p 0}^{(T)} \\
\Delta=\Delta_{0} \\
\Delta^{*}=\Delta_{0}^{*}}
\end{array} \\
& \frac{\delta F_{s}\left[n, \mathbf{j}_{p}^{(T)}, \Delta, \Delta^{*}\right]}{\delta \Delta\left(\mathbf{r} \zeta, \mathbf{r}^{\prime} \zeta^{\prime}\right)} \mid \begin{array}{l}
\substack{n=n_{0} \\
\mathbf{j}_{p}^{(T)}=\mathbf{j}_{p 0}^{(T)} \\
\Delta=\Delta_{0} \\
\Delta^{*}=\Delta_{0}^{*}} \\
\substack{n=n_{0} \\
\mathbf{j}_{p}^{(T)}=\mathbf{j}_{p 0}^{(T)} \\
\Delta=\Delta_{0} \\
\Delta^{*}=\Delta_{0}^{*}}
\end{array} \\
& \left.\frac{\delta F_{s}\left[n, \mathbf{j}_{p}^{(T)}, \Delta, \Delta^{*}\right]}{\delta \Delta^{*}\left(\mathbf{r} \zeta, \mathbf{r}^{\prime} \zeta^{\prime}\right)}\right|_{\substack{n=n_{0} \\
\mathbf{j}_{p}^{(T)}=\mathbf{j}_{p 0}^{(T)} \\
\Delta=\Delta_{0} \\
\Delta^{*}=\Delta_{0}^{*}}}+\left.\frac{\delta F_{x c}\left[n, \mathbf{j}_{p}^{(T)}, \Delta, \Delta^{*}\right]}{\delta \Delta^{*}\left(\mathbf{r} \zeta, \mathbf{r}^{\prime} \zeta^{\prime}\right)}\right|_{\substack{n=n_{0} \\
\mathbf{j}_{p}^{(T)}=\mathbf{j}_{p 0}^{(T)} \\
\Delta=\Delta_{0} \\
\Delta^{*}=\Delta_{0}^{*}}}=0 .
\end{aligned}
$$

Equations (84) - (87) are satisfied by the densities of the variationally-minimum point of the reference system, i.e., $\left(n_{s 0}, \mathbf{j}_{p s 0}^{(T)}, \Delta_{s 0}, \Delta_{s 0}^{*}\right)$, while Eqs. (89) - (92) are satisfied by the correct densities of the real system, i.e., $\left(n_{0}, \mathbf{j}_{p 0}^{(T)}, \Delta_{0}, \Delta_{0}^{*}\right)$. If Eqs. (84) - (87) coincide with Eqs. (89) - (92), respectively, then Eqs. (84) - (87) can be regarded as the equations that are satisfied by the correct densities $\left(n_{0}, \mathbf{j}_{p 0}^{(T)}, \Delta_{0}, \Delta_{0}^{*}\right)$. Specifically the following effective mean-filed potentials can reproduce the correct densities as the densities of the variationally- 
minimum point of the reference system:

$$
\begin{aligned}
& v_{s}(\mathbf{r})=v_{\text {given }}(\mathbf{r})+e^{2} \int \frac{n_{0}\left(\mathbf{r}^{\prime}\right)}{\left|\mathbf{r}-\mathbf{r}^{\prime}\right|} d^{3} r^{\prime}+\left.\frac{\delta F_{x c}\left[n, \mathbf{j}_{p}^{(T)}, \Delta, \Delta^{*}\right]}{\delta n(\mathbf{r})}\right|_{\substack{n=n_{0} \\
j_{p}^{(T)}=j_{p 0}^{(T)} \\
\Delta=\Delta_{0} \\
\Delta^{*}=\Delta_{0}^{*}}}+\frac{e^{2}}{2 m}\left\{\mathbf{A}_{\text {given }}^{2}(\mathbf{r})-\mathbf{A}_{s}^{2}(\mathbf{r})\right\} \\
& \mathbf{A}_{s}(\mathbf{r})=\mathbf{A}_{\text {given }}(\mathbf{r})+\left.\frac{1}{e} \frac{\delta F_{x c}\left[n, \mathbf{j}_{p}^{(T)}, \Delta, \Delta^{*}\right]}{\delta \mathbf{j}_{p}^{(T)}(\mathbf{r})}\right|_{\substack{n=n_{0} \\
j_{p}^{(T)}=j_{p 0}^{(T)}}} \\
& \Delta=\Delta_{0} \\
& \Delta^{*}=\Delta_{0}^{*} \\
& D_{s}^{*}\left(\mathbf{r} \zeta, \mathbf{r}^{\prime} \zeta^{\prime}\right)=\left.\frac{\delta F_{x c}\left[n, \mathbf{j}_{p}^{(T)}, \Delta, \Delta^{*}\right]}{\delta \Delta\left(\mathbf{r} \zeta, \mathbf{r}^{\prime} \zeta^{\prime}\right)}\right|_{\substack{n=n_{0} \\
j_{p}^{(T)}=j_{p 0}^{(T)}}}, \\
& \Delta=\Delta_{0} \\
& \Delta^{*}=\Delta_{0}^{*} \\
& D_{s}\left(\mathbf{r} \zeta, \mathbf{r}^{\prime} \zeta^{\prime}\right)=\left.\frac{\delta F_{x c}\left[n, \mathbf{j}_{p}^{(T)}, \Delta, \Delta^{*}\right]}{\delta \Delta^{*}\left(\mathbf{r} \zeta, \mathbf{r}^{\prime} \zeta^{\prime}\right)}\right|_{\substack{n=n_{0} \\
j_{p}^{(T)}=j_{p 0}^{(T)}}} . \\
& \Delta=\Delta_{0} \\
& \Delta^{*}=\Delta_{0}^{*}
\end{aligned}
$$

The key point to determine the effective mean-field potentials is that the reference system is prepared so that the densities $\left(n_{s 0}, \mathbf{j}_{p s 0}^{(T)}, \Delta_{s 0}, \Delta_{s 0}^{*}\right)$ coincide with the correct densities $\left(n_{0}, \mathbf{j}_{p 0}^{(T)}, \Delta_{0}, \Delta_{0}^{*}\right)$ of the real system.

\section{Basic variables in the reference system}

The basic variables are expressed by using the eigenvalues and eigenfunctions of the BdG-KS equation. By solving the BdG-KS equation, and using the resultant solutions, the Hamiltonian of the reference system is written in the diagonalized form given by Eq. (47). Using Eq. (47), the basic variables of the reference system can be calculated via Eqs. (74) 
and (78) - (81). We have

$$
\begin{aligned}
& n_{0}(\mathbf{r})=\sum_{i} f\left(E_{i}\right) \int\left|u_{i}(\mathbf{r} \zeta)\right|^{2} d \zeta+\sum_{i}\left\{1-f\left(E_{i}\right)\right\} \int\left|v_{i}(\mathbf{r} \zeta)\right|^{2} d \zeta, \\
& \mathbf{j}_{p 0}^{(T)}(\mathbf{r})=\frac{\hbar}{i 4 \pi m} \sum_{i}\left[f\left(E_{i}\right) \int\left\{\nabla_{\mathbf{r}^{\prime}} u_{i}^{*}\left(\mathbf{r}^{\prime} \zeta^{\prime}\right) \times \nabla_{\mathbf{r}^{\prime}} u_{i}\left(\mathbf{r}^{\prime} \zeta^{\prime}\right)\right\} \times \frac{\mathbf{r}-\mathbf{r}^{\prime}}{\left|\mathbf{r}-\mathbf{r}^{\prime}\right|^{3}} d^{3} r^{\prime} d \zeta\right. \\
& \left.\quad+\left\{1-f\left(E_{i}\right)\right\} \int\left\{\nabla_{\mathbf{r}^{\prime}} v_{i}^{*}\left(\mathbf{r}^{\prime} \zeta^{\prime}\right) \times \nabla_{\mathbf{r}^{\prime}} v_{i}\left(\mathbf{r}^{\prime} \zeta^{\prime}\right)\right\} \times \frac{\mathbf{r}-\mathbf{r}^{\prime}}{\left|\mathbf{r}-\mathbf{r}^{\prime}\right|^{3}} d^{3} r^{\prime} d \zeta\right], \\
& \Delta_{0}\left(\mathbf{r} \zeta, \mathbf{r}^{\prime} \zeta^{\prime}\right)=\sum_{i}\left[u_{i}\left(\mathbf{r}^{\prime} \zeta^{\prime}\right) v_{i}(\mathbf{r} \zeta)\left\{1-f\left(E_{i}\right)\right\}+v_{i}\left(\mathbf{r}^{\prime} \zeta^{\prime}\right) u_{i}(\mathbf{r} \zeta) f\left(E_{i}\right)\right], \\
& \Delta_{0}^{*}\left(\mathbf{r} \zeta, \mathbf{r}^{\prime} \zeta^{\prime}\right)=\sum_{i}\left[u_{i}^{*}\left(\mathbf{r}^{\prime} \zeta^{\prime}\right) v_{i}^{*}(\mathbf{r} \zeta)\left\{1-f\left(E_{i}\right)\right\}+v_{i}^{*}\left(\mathbf{r}^{\prime} \zeta^{\prime}\right) u_{i}^{*}(\mathbf{r} \zeta) f\left(E_{i}\right)\right],
\end{aligned}
$$

where $f\left(E_{i}\right)$ is the fermi distribution function given by

$$
f\left(E_{i}\right)=\frac{1}{e^{\beta E_{i}}+1}
$$

\section{Calculation procedure}

The effective mean-field potentials given by Eqs. (93) - (96) are dependent on the correct densities $\left(n_{0}, \mathbf{j}_{p 0}^{(T)}, \Delta_{0}, \Delta_{0}^{*}\right)$, and should be determined in a self-consistent way. The concrete steps of calculating them are as follows: (i) the input set of densities $\left(n_{0}, \mathbf{j}_{p 0}^{(T)}, \Delta_{0}, \Delta_{0}^{*}\right)$ is tentatively given, (ii) using Eqs. (93) - (96), the effective mean-field potentials are calculated, (iii) solving the BdG-KS equation, and substituting these solutions into Eqs. (97) - (100), the new set of densities $\left(n_{0}, \mathbf{j}_{p 0}^{(T)}, \Delta_{0}, \Delta_{0}^{*}\right)$ is obtained, (iv) comparing this set with the input set, and if these are not consistent with each other within some accuracy then the calculations are restarted from (i) with changing the input set. The calculations are repeated until the self-consistency mentioned above is attained.

From the HK theorem, the densities thus obtained correspond to the equilibrium densities for the beforehand-given electromagnetic fields $v_{\text {given }}(\mathbf{r})$ and $\mathbf{A}_{\text {given }}(\mathbf{r})$. The field $v_{\text {given }}(\mathbf{r})$ originates from the charged particles positioned outside the system. On the other hand, $\mathbf{A}_{\text {given }}(\mathbf{r})$ is the potential that electrons of the system feel at the position $\mathbf{r}$, and should be consistent with the densities $n_{0}(\mathbf{r})$ and $\mathbf{j}_{p 0}^{(T)}(\mathbf{r})$. Namely, the beforehand-given field $\mathbf{A}_{\text {given }}(\mathbf{r})$ consists of the field caused by the charged particles positioned outside the system, and the field caused by the charged particles inside the system. The former field is the completely fixed one, while the later field should be determined by using the densities $n_{0}(\mathbf{r})$ and $\mathbf{j}_{p 0}^{(T)}(\mathbf{r})$ 
via the microscopic Maxwell equation $\stackrel{59,60}{ }$. Therefore, in addition to the self-consistent cal-

culation loop for the densities $\left(n_{0}, \mathbf{j}_{p 0}^{(T)}, \Delta_{0}, \Delta_{0}^{*}\right)$, which is explained above, we need further calculation loop to keep the consistency between the beforehand-given electromagnetic field $\mathbf{A}_{\text {given }}(\mathbf{r})$ and the densities $\left(n_{0}(\mathbf{r}), \mathbf{j}_{p 0}^{(T)}(\mathbf{r})\right)$. The concrete steps of the calculations are as follows: (I) $v_{\text {given }}(\mathbf{r})$ is determined in accordance with the charged particles outside the system, while $\mathbf{A}_{\text {given }}(\mathbf{r})$ is tentatively given, (II) using these fields, the corresponding densities $\left(n_{0}, \mathbf{j}_{p 0}^{(T)}, \Delta_{0}, \Delta_{0}^{*}\right)$ are obtained via the calculation loop denoted as (i) - (iv), (III) substituting these densities into the microscopic Maxwell equation $\underline{ }$, the electromagnetic fields caused by the charged particles inside the system are obtained, (IV) adding these fields to the fixed external fields, the new set of the electromagnetic fields are obtained, (V) taking $\mathbf{A}_{\text {given }}(\mathbf{r})$ among the thus-obtained electromagnetic fields, and comparing it with the input one, and if these are not consistent with each other within some accuracy then the calculations are restarted from (I) with changing the input field. The calculations are repeated until the self-consistency is attained.

\section{EXCHANGE-CORRELATION ENERGY FUNCTIONAL}

In the development of the DFT-based or ECS-based theory, the following issues have to be performed. Both of them are indispensable so that the theory works well:

(a) The proof of the HK theorem and the derivation of the KS equation,

(b) Development of the approximate form of the xc energy functional.

We have performed (a) in the preceding sections. In this section, we shall tackle with (b) in two ways. One is the derivation of the rigorous expression for the xc energy functional with the use of the coupling-constant integration. This kind of expression seems to be useful for the development of the approximate forms in the future. Actually, in the conventional $\mathrm{DFT}^{23,24}$, the local density approximation (LDA) for the xc energy functional was developed on the basis of the rigorous expression with the coupling-constant integration 24,62 . The other is a proposal of the practically useful form of the xc energy functional with the use of the approximate solutions of the BdG-KS equation. (Eq. (153)). Specifically, it is devised so that the solution of the BdG-KS equation has the superconducting energy gap which is consistent with the attractive interaction appeared in the Hamiltonian of the real system. 


\section{A. Rigorous expression with the use of the coupling-constant integration}

The Hamiltonian of the real system is given by Eq. (5), while that of the reference system is given by Eq. (66). Here we shall introduce the system which is expressed by the Hamiltonian scaled with the coupling constant $\xi$ :

$$
\hat{H}_{\xi}=\hat{T}+\xi\left(\hat{W}_{1}+\hat{W}_{2}\right)+\hat{U}_{\xi},
$$

where $\hat{U}_{\xi}$ is chosen to be the external potential which makes the equilibrium densities of the system $\hat{H}_{\xi}$ be coincident with the correct densities $\left(n_{0}, \mathbf{j}_{p 0}^{(T)}, \Delta_{0}, \Delta_{0}^{*}\right)$. Let $\hat{U}_{\xi}$ be expressed as

$$
\begin{aligned}
\hat{U}_{\xi}= & \int v_{\xi}(\mathbf{r}) \hat{n}(\mathbf{r}) d^{3} r+e \int \mathbf{A}_{\xi}(\mathbf{r}) \cdot \hat{\mathbf{j}}_{p}^{(T)}(\mathbf{r}) d^{3} r+\frac{e^{2}}{2 m} \int \mathbf{A}_{\xi}(\mathbf{r})^{2} \hat{n}(\mathbf{r}) d^{3} r \\
& +\iint D_{\xi}^{*}\left(\mathbf{r} \zeta, \mathbf{r}^{\prime} \zeta^{\prime}\right) \hat{\Delta}\left(\mathbf{r} \zeta, \mathbf{r}^{\prime} \zeta^{\prime}\right) d^{3} r d \zeta d^{3} r^{\prime} d \zeta^{\prime} \\
& +\iint D_{\xi}\left(\mathbf{r} \zeta, \mathbf{r}^{\prime} \zeta^{\prime}\right) \hat{\Delta}^{\dagger}\left(\mathbf{r} \zeta, \mathbf{r}^{\prime} \zeta^{\prime}\right) d^{3} r d \zeta d^{3} r^{\prime} d \zeta^{\prime}
\end{aligned}
$$

In the special cases of $\xi=1$ and $\xi=0, \hat{U}_{\xi}$ is given by

$$
\hat{U}_{\xi}= \begin{cases}\hat{V}_{1}+\hat{V}_{2}+\hat{V}_{3} & \text { for } \xi=1, \\ \hat{V}_{1 s}+\hat{V}_{2 s}+\hat{V}_{3 s}+\hat{V}_{D s} & \text { for } \xi=0,\end{cases}
$$

respectively. We shall define the following universal energy functional

$$
\begin{aligned}
F_{\xi}\left[n, \mathbf{j}_{p}^{(T)}, \Delta, \Delta^{*}\right]= & \operatorname{Min}_{\hat{\rho} \rightarrow n, \mathbf{j}_{p}^{(T)}, \Delta, \Delta^{*}} \operatorname{Tr}\left[\hat{\rho}\left\{\hat{T}+\xi\left(\hat{W}_{1}+\hat{W}_{2}\right)\right\}+\frac{1}{\beta} \hat{\rho} \ln \hat{\rho}\right] \\
= & \operatorname{Tr}\left[\hat{\rho}_{\xi}\left[n, \mathbf{j}_{p}^{(T)}, \Delta, \Delta^{*}\right]\left\{\hat{T}+\xi\left(\hat{W}_{1}+\hat{W}_{2}\right)\right\}\right. \\
& \left.+\frac{1}{\beta} \hat{\rho}_{\xi}\left[n, \mathbf{j}_{p}^{(T)}, \Delta, \Delta^{*}\right] \ln \hat{\rho}_{\xi}\left[n, \mathbf{j}_{p}^{(T)}, \Delta, \Delta^{*}\right]\right],
\end{aligned}
$$

where the minimizing $\hat{\rho}$ is denoted as $\hat{\rho}_{\xi}\left[n, \mathbf{j}_{p}^{(T)}, \Delta, \Delta^{*}\right]$. Using the fact that $F_{1}\left[n_{0}, \mathbf{j}_{p 0}^{(T)}, \Delta_{0}, \Delta_{0}^{*}\right]$ and $F_{0}\left[n_{0}, \mathbf{j}_{p 0}^{(T)}, \Delta_{0}, \Delta_{0}^{*}\right]$ are coincident with $F\left[n_{0}, \mathbf{j}_{p 0}^{(T)}, \Delta_{0}, \Delta_{0}^{*}\right]$ and $F_{s}\left[n_{0}, \mathbf{j}_{p 0}^{(T)}, \Delta_{0}, \Delta_{0}^{*}\right]$, respectively, the xc energy functional defined by Eq. (88) is rewritten as

$$
F_{x c}\left[n_{0}, \mathbf{j}_{p 0}^{(T)}, \Delta_{0}, \Delta_{0}^{*}\right]=\int_{0}^{1} \frac{d F_{\xi}\left[n_{0}, \mathbf{j}_{p 0}^{(T)}, \Delta_{0}, \Delta_{0}^{*}\right]}{d \xi} d \xi-\frac{e^{2}}{2} \iint \frac{n_{0}(\mathbf{r}) n_{0}\left(\mathbf{r}^{\prime}\right)}{\left|\mathbf{r}-\mathbf{r}^{\prime}\right|} d^{3} r d^{3} r^{\prime} .
$$

Next we shall consider the integrand $d F_{\xi}\left[n_{0}, \mathbf{j}_{p 0}^{(T)}, \Delta_{0}, \Delta_{0}^{*}\right] / d \xi$ in Eq. (106). For the convenience of discussions, the energy functional of the scaled system, which corresponds to 
Eq. (76) of the reference system, is defined as

$$
\begin{aligned}
J_{\xi}^{v_{\xi}-\mu, \mathbf{A}_{\xi}, D_{\xi}, D_{\xi}^{*}}\left[n, \mathbf{j}_{p}^{(T)}, \Delta, \Delta^{*}\right] & =F_{\xi}\left[n, \mathbf{j}_{p}^{(T)}, \Delta, \Delta^{*}\right]+\int\left\{v_{\xi}(\mathbf{r})-\mu\right\} n(\mathbf{r}) d^{3} r \\
& +e \int \mathbf{A}_{\xi}(\mathbf{r}) \cdot \mathbf{j}_{p}^{(T)}(\mathbf{r}) d^{3} r+\frac{e^{2}}{2 m} \int \mathbf{A}_{\xi}(\mathbf{r})^{2} n(\mathbf{r}) d^{3} r \\
& +\iint D_{\xi}^{*}\left(\mathbf{r} \zeta, \mathbf{r}^{\prime} \zeta^{\prime}\right) \Delta\left(\mathbf{r} \zeta, \mathbf{r}^{\prime} \zeta^{\prime}\right) d^{3} r d \zeta d^{3} r^{\prime} d \zeta^{\prime} \\
& +\iint D_{\xi}\left(\mathbf{r} \zeta, \mathbf{r}^{\prime} \zeta^{\prime}\right) \Delta^{*}\left(\mathbf{r} \zeta, \mathbf{r}^{\prime} \zeta^{\prime}\right) d^{3} r d \zeta d^{3} r^{\prime} d \zeta^{\prime}
\end{aligned}
$$

Using Eq. (105), Eq. (107) is rewritten as

$$
\begin{aligned}
J_{\xi}^{v_{\xi}-\mu, \mathbf{A}_{\xi}, D_{\xi}, D_{\xi}^{*}}\left[n, \mathbf{j}_{p}^{(T)}, \Delta, \Delta^{*}\right]= & \operatorname{Tr}\left[\hat{\rho}_{\xi}\left[n, \mathbf{j}_{p}^{(T)}, \Delta, \Delta^{*}\right]\left\{\hat{H}_{\xi}-\mu \hat{N}\right\}\right. \\
& \left.+\frac{1}{\beta} \hat{\rho}_{\xi}\left[n, \mathbf{j}_{p}^{(T)}, \Delta, \Delta^{*}\right] \ln \hat{\rho}_{\xi}\left[n, \mathbf{j}_{p}^{(T)}, \Delta, \Delta^{*}\right]\right] .
\end{aligned}
$$

Differentiating both sides of Eq. (108) with respect to $\xi$, we have

$$
\begin{aligned}
\frac{d}{d \xi} J_{\xi}^{v_{\xi}-\mu, \mathbf{A}_{\xi}, D_{\xi} D_{\xi}^{*}}\left[n, \mathbf{j}_{p}^{(T)}, \Delta, \Delta^{*}\right]= & \operatorname{Tr}\left[\frac{d \hat{\rho}_{\xi}\left[n, \mathbf{j}_{p}^{(T)}, \Delta, \Delta^{*}\right]}{d \xi}\left\{\hat{H}_{\xi}-\mu \hat{N}\right\}\right. \\
& +\hat{\rho}_{\xi}\left[n, \mathbf{j}_{p}^{(T)}, \Delta, \Delta^{*}\right] \frac{d \hat{H}_{\xi}}{d \xi} \\
& +\frac{1}{\beta} \frac{d \hat{\rho}_{\xi}\left[n, \mathbf{j}_{p}^{(T)}, \Delta, \Delta^{*}\right]}{d \xi} \ln \hat{\rho}_{\xi}\left[n, \mathbf{j}_{p}^{(T)}, \Delta, \Delta^{*}\right] \\
& \left.+\frac{1}{\beta} \hat{\rho}_{\xi}\left[n, \mathbf{j}_{p}^{(T)}, \Delta, \Delta^{*}\right] \frac{d}{d \xi} \ln \hat{\rho}_{\xi}\left[n, \mathbf{j}_{p}^{(T)}, \Delta, \Delta^{*}\right]\right] .
\end{aligned}
$$

Of course, Eq. (109) holds also for the correct densities $\left(n_{0}, \mathbf{j}_{p 0}^{(T)}, \Delta_{0}, \Delta_{0}^{*}\right)$. In that case, we can use the HK theorem for the scaled system, the result of which gives the relation such that $\hat{\rho}_{\xi}\left[n_{0}, \mathbf{j}_{p 0}^{(T)}, \Delta_{0}, \Delta_{0}^{*}\right]=e^{-\beta\left(\hat{H}_{\xi}-\mu \hat{N}\right)} / \Xi_{\xi}$ with $\Xi_{\xi}=\operatorname{Tr}\left\{e^{-\beta\left(\hat{H}_{\xi}-\mu \hat{N}\right)}\right\}$. Substituting this relation into Eq. (109), we have

$$
\frac{d}{d \xi} J_{\xi}^{v_{\xi}-\mu, \mathbf{A}_{\xi}, D_{\xi}, D_{\xi}^{*}}\left[n_{0}, \mathbf{j}_{p 0}^{(T)}, \Delta_{0}, \Delta_{0}^{*}\right]=\operatorname{Tr}\left\{\hat{\rho}_{\xi}\left[n_{0}, \mathbf{j}_{p 0}^{(T)}, \Delta_{0}, \Delta_{0}^{*}\right]\left(\hat{W}_{1}+\hat{W}_{2}+\frac{d \hat{U}_{\xi}}{d \xi}\right)\right\} .
$$

Considering the explicit form of $J_{\xi}^{v_{\xi}-\mu, \mathbf{A}_{\xi}, D_{\xi}, D_{\xi}^{*}}\left[n, \mathbf{j}_{p}^{(T)}, \Delta, \Delta^{*}\right]$ given by Eq. (108), Eq. (110) can be regarded as the Hellman-Feynman theorem at the finite temperature. Namely, the derivative of the statistical operator with respect to $\xi$ does not appear but the derivative of the energy operators with respect to $\xi$ appears in the RHS of Eq. (110). 
On the other hand, using Eq. (107), we have

$$
\begin{aligned}
& \frac{d}{d \xi} J_{\xi}^{v_{\xi}-\mu, \mathbf{A}_{\xi}, D_{\xi}, D_{\xi}^{*}}\left[n_{0}, \mathbf{j}_{p 0}^{(T)}, \Delta_{0}, \Delta_{0}^{*}\right]=\frac{d F_{\xi}\left[n_{0}, \mathbf{j}_{p 0}^{(T)}, \Delta_{0}, \Delta_{0}^{*}\right]_{\xi}}{d \xi} \\
& +\operatorname{Tr}\left\{\hat{\rho}_{\xi}\left[n_{0}, \mathbf{j}_{p 0}^{(T)}, \Delta_{0}, \Delta_{0}^{*}\right] \frac{d \hat{U}_{\xi}}{d \xi}\right\}
\end{aligned}
$$

Comparing Eq. (110) with Eq. (111), the expression for $d F_{\xi}\left[n_{0}, \mathbf{j}_{p 0}^{(T)}, \Delta_{0}, \Delta_{0}^{*}\right] / d \xi$ is obtained. Substituting it into Eq. (106) yields

$$
\begin{aligned}
F_{x c}\left[n_{0}, \mathbf{j}_{p 0}^{(T)}, \Delta_{0}, \Delta_{0}^{*}\right]= & \int_{0}^{1} \operatorname{Tr}\left\{\hat{\rho}_{\xi}\left[n_{0}, \mathbf{j}_{p 0}^{(T)}, \Delta_{0}, \Delta_{0}^{*}\right]\left(\hat{W}_{1}+\hat{W}_{2}\right)\right\} d \xi \\
& -\frac{e^{2}}{2} \iint \frac{n_{0}(\mathbf{r}) n_{0}\left(\mathbf{r}^{\prime}\right)}{\left|\mathbf{r}-\mathbf{r}^{\prime}\right|} d^{3} r d^{3} r^{\prime} .
\end{aligned}
$$

Equation (112) is exactly the rigorous expression for the xc energy functional of the present theory.

Let us consider the meaning of the coupling-constant integration in Eq. (112). Rewriting the xc energy functional defined by Eq. (88) by means of the minimizing statistical operators, we have

$$
\begin{aligned}
F_{x c}\left[n_{0}, \mathbf{j}_{p 0}^{(T)}, \Delta_{0}, \Delta_{0}^{*}\right] & =\operatorname{Tr}\left\{\hat{\rho}_{\min }\left[n_{0}, \mathbf{j}_{p 0}^{(T)}, \Delta_{0}, \Delta_{0}^{*}\right]\left(\hat{W}_{1}+\hat{W}_{2}\right)\right\}-\frac{e^{2}}{2} \iint \frac{n_{0}(\mathbf{r}) n_{0}\left(\mathbf{r}^{\prime}\right)}{\left|\mathbf{r}-\mathbf{r}^{\prime}\right|} d^{3} r d^{3} r^{\prime} \\
& +\operatorname{Tr}\left\{\hat{\rho}_{\min }\left[n_{0}, \mathbf{j}_{p 0}^{(T)}, \Delta_{0}, \Delta_{0}^{*}\right] \hat{T}\right\}-\operatorname{Tr}\left\{\hat{\rho}_{s, \min }\left[n_{0}, \mathbf{j}_{p 0}^{(T)}, \Delta_{0}, \Delta_{0}^{*}\right] \hat{T}\right\} \\
& +\frac{1}{\beta} \operatorname{Tr}\left\{\hat{\rho}_{\min }\left[n_{0}, \mathbf{j}_{p 0}^{(T)}, \Delta_{0}, \Delta_{0}^{*}\right] \ln \hat{\rho}_{\min }\left[n_{0}, \mathbf{j}_{p 0}^{(T)}, \Delta_{0}, \Delta_{0}^{*}\right]\right\} \\
& -\frac{1}{\beta} \operatorname{Tr}\left\{\hat{\rho}_{s, \min }\left[n_{0}, \mathbf{j}_{p 0}^{(T)}, \Delta_{0}, \Delta_{0}^{*}\right] \ln \hat{\rho}_{s, \min }\left[n_{0}, \mathbf{j}_{p 0}^{(T)}, \Delta_{0}, \Delta_{0}^{*}\right]\right\}, \quad(113)
\end{aligned}
$$

where the first and second terms of the RHS correspond to the xc energy, and the third and fourth terms mean the difference of the kinetic energy between the real and reference systems, and the fifth and sixth terms mean the difference of the entropy between these systems. Comparing this result with Eq. (112), the xc energy functional of the CDFT for the superconductor contains not only the xc energy but also the difference of the kinetic energy and that of the entropy via the coupling-constant integration ${ }^{63}$.

\section{B. An approximate form of the xc energy functional}

In this subsection, we present the approximate forms of $F_{x c}\left[n, \mathbf{j}_{p}^{(T)}, \Delta, \Delta^{*}\right]$ along the following procedure. (i) First, the approximate form of the effective pair potential which 
induces the OPSS with the spin-singlet or spin-triplet symmetry is proposed. (ii) Using such a potential, we derive the approximate solution of the BdG-KS equation given by Eqs. (53) and (93) - (96). The resultant solution of the energy spectrum possesses the energy gap for the excitation, which seems to be physically reasonable for the superconducting state. (iii) Finally, we derive the xc energy functional that yields the above-mentioned effective pair potential. It is shown that such an approximate form is related to the attractive interaction that is included in the Hamiltonian Eq. (5). In what follows, we shall show the details.

Step (i)

Suppose that the effective pair potential that induces the OPSS with the spin symmetry $\tau$ is denoted as $D_{s, \tau}\left(\mathbf{r} \zeta, \mathbf{r}^{\prime} \zeta^{\prime}\right)$. Here the spin wave functions of the first, second and third terms of Eq. (3), which are the spin-triplet wave functions, are labeled as $\tau_{1}, \tau_{2}, \tau_{3}$, respectively, and the spin wave function of the fourth term of Eq. (3), which is the spin-singlet one, is labeled as $\tau_{4} . D_{s, \tau}\left(\mathbf{r} \zeta, \mathbf{r}^{\prime} \zeta^{\prime}\right)$ is assumed to be split into the spin-dependent and spatialdependent parts as follows:

$$
D_{s, \tau}\left(\mathbf{r} \zeta, \mathbf{r}^{\prime} \zeta^{\prime}\right)=\frac{1}{2} D_{s 1, \tau}\left(\zeta, \zeta^{\prime}\right) D_{s 2, \tau}\left(\mathbf{r}, \mathbf{r}^{\prime}\right)
$$

Let us consider the concrete forms of $D_{s 1, \tau}\left(\zeta, \zeta^{\prime}\right)$ and $D_{s 2, \tau}\left(\mathbf{r}, \mathbf{r}^{\prime}\right)$ which induce the OPSS in the reference system. The OPSS given by Eq. (3) is rewritten in the following form:

$$
\hat{\Delta}\left(\mathbf{r} \zeta, \mathbf{r}^{\prime} \zeta^{\prime}\right)=\sum_{\tau} \sum_{k_{i}} \sum_{k_{j}} C_{k_{i} \sigma_{\tau}} C_{k_{j} \sigma_{\tau}^{\prime}} \Psi_{\tau}^{k_{i} k_{j}}\left(\mathbf{r}, \mathbf{r}^{\prime}\right) \Phi_{\tau}\left(\zeta, \zeta^{\prime}\right)
$$

where $\Psi_{\tau}^{k_{i} k_{j}}\left(\mathbf{r}, \mathbf{r}^{\prime}\right)$ and $\Phi_{\tau}\left(\zeta, \zeta^{\prime}\right)$ are the spatial wave function and spin one for the OPSS with the spin symmetry $\tau$, respectively. Substituting Eqs. (114) and (115) into the third term of Eq. (43), the integrals which are dependent on the spin wave function and spatial wave function are, respectively, expressed as

$$
\iint D_{s 1, \tau}^{*}\left(\zeta, \zeta^{\prime}\right) \Phi_{\tau}\left(\zeta, \zeta^{\prime}\right) d \zeta d \zeta^{\prime}
$$

and

$$
\iint D_{s 2, \tau}^{*}\left(\mathbf{r}, \mathbf{r}^{\prime}\right) \Psi_{\tau}^{k_{i} k_{j}}\left(\mathbf{r}, \mathbf{r}^{\prime}\right) d^{3} r d^{3} r^{\prime}
$$


As an illustrative form of $D_{s 1, \tau}\left(\zeta, \zeta^{\prime}\right)$ that gives the nonzero value of Eq. (116), we present

$$
D_{s 1, \tau}\left(\zeta, \zeta^{\prime}\right)= \begin{cases}\frac{1}{2}\left(\delta_{\zeta \zeta^{\prime}}+\left\langle\zeta\left|\sigma_{z}\right| \zeta\right\rangle\right) & \text { for } \tau=\tau_{1}, \\ \frac{1}{2}\left(\delta_{\zeta \zeta^{\prime}}-\left\langle\zeta\left|\sigma_{z}\right| \zeta\right\rangle\right) & \text { for } \tau=\tau_{2}, \\ \left\langle\zeta\left|\sigma_{x}\right| \zeta\right\rangle & \text { for } \tau=\tau_{3}, \\ \left\langle\zeta\left|\sigma_{y}\right| \zeta\right\rangle & \text { for } \tau=\tau_{4},\end{cases}
$$

where $\sigma_{x}, \sigma_{y}$ and $\sigma_{z}$ are the Pauli marices.

On the other hand, in order to make Eq. (117) not be zero, the spatial-dependent part $D_{s 2, \tau}\left(\mathbf{r}, \mathbf{r}^{\prime}\right)$ needs to possess the following properties:

$$
\begin{array}{ll}
D_{s 2, \tau}\left(\mathbf{r}, \mathbf{r}^{\prime}\right)-D_{s 2, \tau}\left(\mathbf{r}^{\prime}, \mathbf{r}\right) \neq 0 & \text { for } \tau=\tau_{1}, \tau_{2}, \tau_{3}, \\
D_{s 2, \tau}\left(\mathbf{r}, \mathbf{r}^{\prime}\right)+D_{s 2, \tau}\left(\mathbf{r}^{\prime}, \mathbf{r}\right) \neq 0 & \text { for } \tau=\tau_{4},
\end{array}
$$

because $\Psi_{\tau}^{k_{i} k_{j}}\left(\mathbf{r}, \mathbf{r}^{\prime}\right)$ is the antisymmetric function with respect to the permutation of the spatial coordinates in the cases of $\tau=\tau_{1}, \tau_{2}, \tau_{3}$, while it is the symmetric function in the case of $\tau=\tau_{4}$. For the latter convenience, we define the function $d_{s 2, \tau}\left(\mathbf{r}, \mathbf{r}^{\prime}\right)$ as follows:

$$
d_{s 2, \tau}\left(\mathbf{r}, \mathbf{r}^{\prime}\right)= \begin{cases}\frac{1}{2}\left\{D_{s 2, \tau}\left(\mathbf{r}, \mathbf{r}^{\prime}\right)-D_{s 2, \tau}\left(\mathbf{r}^{\prime}, \mathbf{r}\right)\right\} & \text { for } \tau_{1}, \tau_{2}, \tau_{3}, \\ \frac{1}{2}\left\{D_{s 2, \tau}\left(\mathbf{r}, \mathbf{r}^{\prime}\right)+D_{s 2, \tau}\left(\mathbf{r}^{\prime}, \mathbf{r}\right)\right\} & \text { for } \tau=\tau_{4} .\end{cases}
$$

As can be seen in Eq. (53), the effective pair potential is included in a form of Eq. (54). Using Eq. (120), and considering the symmetry of Eq. (118), Eq. (54) is rewritten as

$$
\tilde{D}_{s, \tau}\left(\mathbf{r} \zeta, \mathbf{r}^{\prime} \zeta^{\prime}\right)=D_{s 1, \tau}\left(\zeta, \zeta^{\prime}\right) d_{s 2, \tau}\left(\mathbf{r}, \mathbf{r}^{\prime}\right) .
$$

Step (ii)

Using Eq. (121), let us consider the approximate solution of Eq. (53). We adopt the approximation method presented by P. G. de Gennes $\underline{\underline{48}}$. Specifically, the solution of the normal state is modified to be suitable for the superconducting state ${ }^{\underline{48}}$. The solution of the normal state obeys the equation

$$
\left(h_{s}^{\mathbf{r}}-\mu\right) w_{i}(\mathbf{r})=\varepsilon_{i} w_{i}(\mathbf{r}),
$$

where $h_{s}^{\mathrm{r}}$ is given by Eq. (52). In the case of the spin-singlet $\left(\tau=\tau_{4}\right)$, the solution of Eq. (53) is searched in the following form $\underline{48}$ :

$$
\begin{aligned}
& u_{i}(\mathbf{r} \zeta)=\bar{u}_{i} \chi_{u_{i}}(\zeta) w_{i}(\mathbf{r}), \\
& v_{i}(\mathbf{r} \zeta)=\bar{v}_{i} \chi_{v_{i}}(\zeta) w_{i}(\mathbf{r}),
\end{aligned}
$$


where $\bar{u}_{i}$ and $\bar{v}_{i}$ are complex numbers. In order to satisfy the orthonormality of the solutions of the BdG-KS equation (Sec. IV C), we suppose that

$$
\begin{aligned}
& \int \chi_{u_{i}}(\zeta) \chi_{v_{i}}(\zeta) d \zeta=0, \\
& \left|\bar{u}_{i}\right|^{2}+\left|\bar{v}_{i}\right|^{2}=1 .
\end{aligned}
$$

These relations guarantee Eqs. (62), (64) and (65) to hold. Substituting Eq. (123) into Eq. (53), we can easily obtain

$$
\left(\begin{array}{cc}
\varepsilon_{i}-E_{i} & \int \chi_{u_{i}}(\zeta) D_{s 1, \tau_{4}}\left(\zeta, \zeta^{\prime}\right) \chi_{v_{i}}\left(\zeta^{\prime}\right) d \zeta d \zeta^{\prime} \\
-\int \chi_{v_{i}}(\zeta) D_{s 1, \tau_{4}}^{*}\left(\zeta, \zeta^{\prime}\right) \chi_{u_{i}}\left(\zeta^{\prime}\right) d \zeta d \zeta^{\prime} & \times \int w_{i}^{*}(\mathbf{r}) d_{s 2, \tau_{4}}\left(\mathbf{r}, \mathbf{r}^{\prime}\right) w_{i}^{*}\left(\mathbf{r}^{\prime}\right) d^{3} r d^{3} r^{\prime} \\
\times \int w_{i}(\mathbf{r}) d_{s 2, \tau_{4}}^{*}\left(\mathbf{r}, \mathbf{r}^{\prime}\right) w_{i}\left(\mathbf{r}^{\prime}\right) d^{3} r d^{3} r^{\prime} & -\left(\varepsilon_{i}+E_{i}\right)
\end{array}\right)\left(\begin{array}{c}
\bar{u}_{i} \\
\bar{v}_{i}^{*}
\end{array}\right)=\left(\begin{array}{l}
0 \\
0
\end{array}\right)
$$

which yields

$$
\begin{aligned}
E_{i}^{2}=\varepsilon_{i}^{2}- & \int \chi_{u_{i}}(\zeta) D_{s 1, \tau_{4}}\left(\zeta, \zeta^{\prime}\right) \chi_{v_{i}}(\zeta) d \zeta d \zeta^{\prime} \int \chi_{v_{i}}(\zeta) D_{s 1, \tau_{4}}^{*}\left(\zeta, \zeta^{\prime}\right) \chi_{u_{i}}(\zeta) d \zeta d \zeta^{\prime} \\
& \times\left|\int w_{i}^{*}(\mathbf{r}) d_{s 2, \tau_{4}}\left(\mathbf{r}, \mathbf{r}^{\prime}\right) w_{i}^{*}\left(\mathbf{r}^{\prime}\right) d^{3} r d^{3} r^{\prime}\right|^{2}
\end{aligned}
$$

Substituting Eq. (118) into Eq. (127), and if we use $\chi_{u_{i}}(\zeta)=\chi_{\uparrow}(\zeta), \quad \chi_{v_{i}}(\zeta)=\chi_{\downarrow}(\zeta)$ as an example that is satisfied with Eq. (124), then Eq. (127) becomes

$$
E_{i}^{2}=\varepsilon_{i}^{2}+\left|D_{i}^{\text {singlet }}\right|^{2} \quad \text { with } \quad D_{i}^{\text {singlet }}=\int w_{i}^{*}(\mathbf{r}) d_{s 2, \tau_{4}}\left(\mathbf{r}, \mathbf{r}^{\prime}\right) w_{i}^{*}\left(\mathbf{r}^{\prime}\right) d^{3} r d^{3} r^{\prime}
$$

Since the energy gap appears in the energy spectrum of the superconducting state, the result Eq. (128) seems to be physically reasonable ${ }^{48}$.

Next we consider the case of the spin-triplet $\left(\tau=\tau_{1}, \tau_{2}, \tau_{3}\right)$. Instead of Eq. (123), we use

$$
\begin{aligned}
& u_{i}(\mathbf{r} \zeta)=\bar{u}_{i} \chi_{u_{i}}(\zeta) x_{i}(\mathbf{r}), \\
& v_{i}(\mathbf{r} \zeta)=\bar{v}_{i} \chi_{v_{i}}(\zeta) y_{i}(\mathbf{r}),
\end{aligned}
$$

where $x_{i}(\mathbf{r})$ and $y_{i}(\mathbf{r})$ are the solutions of Eq. (122), and the corresponding energies $\kappa_{i}$ and $\lambda_{i}$ are different from each other, which implies the orthogonality between $x_{i}(\mathbf{r})$ and $y_{i}(\mathbf{r})$. 
This also guarantees Eqs. (62), (64) and (65). Substituting Eq. (129) into Eq. (53), we get

$$
\left(\begin{array}{cc}
\kappa_{i}-E_{i} & \int \chi_{u_{i}}(\zeta) D_{s 1, \tau}\left(\zeta, \zeta^{\prime}\right) \chi_{v_{i}}\left(\zeta^{\prime}\right) d \zeta d \zeta^{\prime} \\
-\int \chi_{v_{i}}(\zeta) D_{s 1, \tau}^{*}\left(\zeta, \zeta^{\prime}\right) \chi_{u_{i}}\left(\zeta^{\prime}\right) d \zeta d \zeta^{\prime} & \times \int x_{i}^{*}(\mathbf{r}) d_{s 2, \tau}\left(\mathbf{r}, \mathbf{r}^{\prime}\right) y_{i}^{*}\left(\mathbf{r}^{\prime}\right) d^{3} r d^{3} r^{\prime} \\
\times \int y_{i}(\mathbf{r}) d_{s 2, \tau}^{*}\left(\mathbf{r}, \mathbf{r}^{\prime}\right) x_{i}\left(\mathbf{r}^{\prime}\right) d^{3} r d^{3} r^{\prime} & -\left(\lambda_{i}+E_{i}\right)
\end{array}\right)\left(\begin{array}{l}
\bar{u}_{i} \\
\bar{v}_{i}^{*}
\end{array}\right)=\left(\begin{array}{l}
0 \\
0
\end{array}\right),
$$

which immediately leads to

$$
\begin{aligned}
E_{i}^{2}+E_{i}\left(\lambda_{i}-\kappa_{i}\right)-\lambda_{i} \kappa_{i}- & \int \chi_{u_{i}}(\zeta) D_{s 1, \tau}\left(\zeta, \zeta^{\prime}\right) \chi_{v_{i}}\left(\zeta^{\prime}\right) d \zeta d \zeta^{\prime} \int \chi_{v_{i}}(\zeta) D_{s 1, \tau}^{*}\left(\zeta, \zeta^{\prime}\right) \chi_{u_{i}}\left(\zeta^{\prime}\right) d \zeta d \zeta^{\prime} \\
& \times\left|\int y_{i}^{*}(\mathbf{r}) d_{s 2, \tau}\left(\mathbf{r}, \mathbf{r}^{\prime}\right) x_{i}^{*}\left(\mathbf{r}^{\prime}\right) d^{3} r d^{3} r^{\prime}\right|^{2}=0
\end{aligned}
$$

where $\tau=\tau_{1}, \tau_{2}, \tau_{3}$, and Eq. (120) is used. Hereafter, we shall deal with the case of $\tau=\tau_{1}$ as an example for the spin-triplet case. Substituting Eq. (118) for the case of $\tau=\tau_{1}$ into Eq. (131), and if we use $\chi_{u_{i}}(\zeta)=\chi_{\uparrow}(\zeta), \chi_{v_{i}}(\zeta)=\chi_{\uparrow}(\zeta)$, then Eq. (131) becomes

$$
E_{i}^{2}+E_{i}\left(\lambda_{i}-\kappa_{i}\right)-\lambda_{i} \kappa_{i}-\left|D_{i}^{\text {triplet }}\right|^{2}=0 \quad \text { with } \quad D_{i}^{\text {triplet }}=\int y_{i}^{*}(\mathbf{r}) d_{s 2, \tau_{1}}\left(\mathbf{r}, \mathbf{r}^{\prime}\right) x_{i}^{*}\left(\mathbf{r}^{\prime}\right) d^{3} r d^{3} r^{\prime} .
$$

If we choose $x_{i}(\mathbf{r})$ and $y_{i}(\mathbf{r})$ such that the corresponding energies $\kappa_{i}$ and $\lambda_{i}$ are close to each other, i.e., $\kappa_{i} \approx \lambda_{i}$, then Eq. (132) approximately becomes to

$$
E_{i}^{2} \approx \kappa_{i}^{2}+\left|D_{i}^{\text {triplet }}\right|^{2}
$$

Similarly to the case of the spin-singlet, this result physically sounds reasonable due to the appearance of the energy gap. Note that choosing the normal states which satisfy $\kappa_{i} \neq \lambda_{i}$ but $\kappa_{i} \approx \lambda_{i}$ is easy in metals because the density of states near the Fermi level is generally high in metals.

\section{Step (iii)}

Thus, the approximate forms of the effective pair potential lead to the energy spectrum accompanied by the energy gap for excitations, i.e. Eqs. (128) and (133). Next let us propose the illustrative forms of $D_{s 2, \tau}\left(\mathbf{r}, \mathbf{r}^{\prime}\right)$ ( or $\left.d_{s 2, \tau}\left(\mathbf{r}, \mathbf{r}^{\prime}\right)\right)$ appeared in Eqs. (128) and (133) with the aid of the spirit of the LDA of the DFT 24 . After that, using these functions, we finally present the approximate expression for $F_{x c}\left[n, \mathbf{j}_{p}^{(T)}, \Delta, \Delta^{*}\right]$ that yields the effective pair potential $D_{s, \tau}\left(\mathbf{r} \zeta, \mathbf{r}^{\prime} \zeta^{\prime}\right)$ properly. 
The LDA of the DFT borrows the results of the exchange and correlation energies of the homogeneous electron liquid $\underline{64}$. The LDA expression for the xc energy functional is correct in the limiting system such as the homogeneous electron system. With reference to the manner of the LDA, we propose the illustrative form of the effective pair potential. Specifically, the effective pair potential is determined by requiring that the energy gap given by Eq. (128) or (133) coincides with the correct one in the limiting system where the results of the BCS theory are sufficiently satisfactory ${ }^{53}$. If the actual system is not far from such a limiting system, then it is expected that the approximation mentioned above works well. This expectation is also the same as that of the LDA in the DFT르.

With this approximation the energy gap is identical with that of the BCS theory. In the case of the spin-singlet, we have

$$
\Gamma_{\mathrm{BCS}}^{2}=\left|D_{i}^{\text {singlet }}\right|^{2}=\left|\int w_{i}^{*}(\mathbf{r}) d_{s 2, \tau_{4}}\left(\mathbf{r}, \mathbf{r}^{\prime}\right) w_{i}^{*}\left(\mathbf{r}^{\prime}\right) d^{3} r d^{3} r^{\prime}\right|^{2},
$$

where $\Gamma_{B C S}$ is the energy gap of the BCS theory that is given by ${ }^{53}$

$$
\Gamma_{\mathrm{BCS}}=2 \hbar \omega_{D} e^{-\frac{1}{V_{0} N(0)}}
$$

where $\omega_{D}$ and $N(0)$ are the Debye frequency and the density of states at the Fermi level, respectively, and where $V_{0}$ is the attractive interaction made by the approximation such that $V_{\mathbf{k k}^{\prime}}$ appeared in Eq. (14) is simplified into $-V_{0}$ for the electrons near the Fermi level ${ }^{53}$. As a concrete form of $D_{s 2, \tau_{4}}\left(\mathbf{r}, \mathbf{r}^{\prime}\right)$ which meets Eq. (134), we can choose

$$
D_{s 2, \tau_{4}}\left(\mathbf{r}, \mathbf{r}^{\prime}\right)=w_{i}(\mathbf{r}) w_{i}\left(\mathbf{r}^{\prime}\right)\left\{2 \hbar \omega_{D} e^{-\frac{1}{V_{0} N(0)}}\right\}
$$

where Eq. (120) is used.

Next we consider the case of the spin-triplet $\left(\tau=\tau_{1}\right)$. It is assumed that the relation between the energy gap $\Gamma_{\text {triplet }}$ and attractive interaction $V_{0}$ is denoted as $\Gamma_{\text {triplet }}\left(V_{0}\right)$ instead of Eq. (135). Similarly to Eq. (134), we use the following approximation:

$$
\Gamma_{\text {triplet }}\left(V_{0}\right)^{2}=\left|D_{i}^{\text {triplet }}\right|^{2}=\left|\int y_{i}^{*}(\mathbf{r}) d_{s 2, \tau_{1}}\left(\mathbf{r}, \mathbf{r}^{\prime}\right) x_{i}^{*}\left(\mathbf{r}^{\prime}\right) d^{3} r d^{3} r^{\prime}\right|^{2} \text {. }
$$

As a concrete form of $D_{s 2, \tau_{1}}\left(\mathbf{r}, \mathbf{r}^{\prime}\right)$ which meets Eq. (137), we can choose

$$
D_{s 2, \tau}\left(\mathbf{r}, \mathbf{r}^{\prime}\right)=\left\{y_{i}(\mathbf{r}) x_{i}\left(\mathbf{r}^{\prime}\right)-y_{i}\left(\mathbf{r}^{\prime}\right) x_{i}(\mathbf{r})\right\} \Gamma_{\text {triplet }}\left(V_{0}\right),
$$


where the orthogonality between $x_{i}(\mathbf{r})$ and $y_{i}(\mathbf{r})$ and Eq. (120) are used. Thus, the illustrative forms of $D_{s 2, \tau_{1}}\left(\mathbf{r}, \mathbf{r}^{\prime}\right)$ are obtained, i.e., Eq. (136) for the spin-singlet case and Eq. (138) for the spin-triplet case.

Finally let us consider the approximate expression for $F_{x c}\left[n, \mathbf{j}_{p}^{(T)}, \Delta, \Delta^{*}\right]$. Suppose that $F_{x c}\left[n, \mathbf{j}_{p}^{(T)}, \Delta, \Delta^{*}\right]$ is decomposed into two parts, i.e., the OPSS-dependent part $E_{x c}^{S}\left[n, \mathbf{j}_{p}^{(T)}, \Delta, \Delta^{*}\right]$ and the OPSS-independent part $E_{x c}^{N}\left[n, \mathbf{j}_{p}^{(T)}\right]$. Namely we have

$$
F_{x c}\left[n, \mathbf{j}_{p}^{(T)}, \Delta, \Delta^{*}\right]=E_{x c}^{S}\left[n, \mathbf{j}_{p}^{(T)}, \Delta, \Delta^{*}\right]+E_{x c}^{N}\left[n, \mathbf{j}_{p}^{(T)}\right] .
$$

Aiming at developing the approximate form that is effective for the small magnitude of the OPSS, we will take only the first-order term in the expansion of $E_{x c}^{S}\left[n, \mathbf{j}_{p}^{(T)}, \Delta, \Delta^{*}\right]$ with respect to the OPSS. That is to say,

$$
\begin{aligned}
E_{x c}^{S}\left[n, \mathbf{j}_{p}^{(T)}, \Delta, \Delta^{*}\right] & =\frac{1}{2} \int D_{s 1, \tau}\left(\zeta, \zeta^{\prime}\right) D_{s 2, \tau}\left(\mathbf{r}, \mathbf{r}^{\prime}\right) \Delta^{*}\left(\mathbf{r} \zeta, \mathbf{r}^{\prime} \zeta^{\prime}\right) d^{3} r d \zeta d^{3} r^{\prime} d \zeta^{\prime} \\
& +\frac{1}{2} \int D_{s 1, \tau}^{*}\left(\zeta, \zeta^{\prime}\right) D_{s 2, \tau}^{*}\left(\mathbf{r}, \mathbf{r}^{\prime}\right) \Delta\left(\mathbf{r} \zeta, \mathbf{r}^{\prime} \zeta^{\prime}\right) d^{3} r d \zeta d^{3} r^{\prime} d \zeta^{\prime}
\end{aligned}
$$

where Eq. (140) is consistently satisfied with Eqs. (95) and (96) if $D_{s 2, \tau}\left(\mathbf{r}, \mathbf{r}^{\prime}\right)$ is supposed to be independent of the OPSS. As can be seen in Eqs. (136) and (138), $D_{s 2, \tau}\left(\mathbf{r}, \mathbf{r}^{\prime}\right)$ is written by means of the wave functions of the normal state. Although they are formally dependent on the OPSS through the effective mean-field potentials $v_{s}(\mathbf{r})$ and $\mathbf{A}_{s}(\mathbf{r})$, the wave functions of the normal state may be approximately calculated by utilizing the xc energy functional which does not depend on the OPSS. This is because the electronic structures of the normal state have been usually and successfully calculated by means of the xc energy functional without the OPSS dependence ${ }^{65}$.

When the effective pair potentials become zero, namely, when the system is in the normal state ${ }^{\underline{66}}$, Eq. (140) becomes zero and the resulting $F_{x c}\left[n, \mathbf{j}_{p}^{(T)}, \Delta, \Delta^{*}\right]$ is equal to $E_{x c}^{N}\left[n, \mathbf{j}_{p}^{(T)}\right]$ in Eq. (139)). Therefore, $E_{x c}^{N}\left[n, \mathbf{j}_{p}^{(T)}\right]$ can be regarded as the xc energy functional of the normal state. As an approximate form, the vorticity expansion approximation (VEA) of the CDFT, which has been previously developed $\underline{\underline{43}} \underline{\underline{67}} \underline{\underline{70}}$, would be available and useful.

Substituting Eqs. (118) and (136) into Eq. (140) and, for example, using the VEA

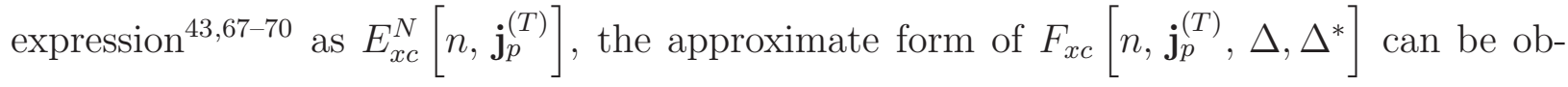
tained for the case of the spin-singlet. On the other hand, substituting Eqs. (118) and (138) into Eq. (140), the approximate form of the xc energy functional for the case of 
the spin-triplet $\left(\tau=\tau_{1}\right)$ can be obtained. It should be noted that the attractive interaction $V_{0}$ which is originally contained in the Hamiltonian Eq. (5) is explicitly contained in

$F_{x c}\left[n, \mathbf{j}_{p}^{(T)}, \Delta, \Delta^{*}\right]$. This is an advisable feature for the xc energy functional because it intrinsically includes the quantum effects of the electron-electron interaction.

\section{CONCLUDING REMARKS}

In this paper, we present the CDFT for the superconductor with the improvements on several issues listed as (i) - (iv) in Sec. I. They are all indispensable for constructing the CDFT which is suitable for superconductors, though such improvements have not sufficiently been performed in the previous works $\underline{26}, \underline{27}, \underline{45}$. The present CDFT reproduces the equilibrium values of the OPSS, electron density and transverse component of the paramagnetic currentdensity for the superconductor immersed in the magnetic field. In what follows, we shall discuss the features of the present CDFT.

(A) As mentioned in Sec. II, the OPSS contains the information on the pairing states of the superconducting state. Since the OPSS is directly evaluated by the present CDFT, various kinds of superconducting properties can be discussed.

(A-1) Not only the critical temperature but also the critical magnetic field can be predicted by evaluating the dependences of the OPSS on the temperature and on the external magnetic field. The temperature and magnetic field in the case when the OPSS disappears correspond to the critical temperature and critical magnetic field, respectively.

(A-2) The spin symmetry for the pairing state is understood from the OPSS directly. It can be verified from Eq. (3) whether the pairing state has as the spin part the spin-singlet symmetry or spin-triplet one or their mixed one.

(A-3) The spatial dependence of the OPSS explicitly shows the spatial symmetry and distribution of the pairing state. The dependence of the OPSS on the relative coordinates gives the spatial broadening of the individual pairing state. It clarifies to what extent the pairing state is close to the Bose particle. On the other hand, the dependence of the OPSS on the coordinates of the center of gravity gives the spatial distribution of the superconducting phase. The disappearing regions of the OPSS can be regarded as the spatial pattern of the magnetic vortex in the mixed state of the Type-II superconductor.

(B) In addition to the OPSS, we can obtain the current-density in the present CDFT. 
This is a striking feature of this theory because the physical phenomena which are related to the current-density can be discussed directly. Specifically, we can discuss the Meissner effect, Silsbee's rule that gives the relation between the critical current-density and the critical magnetic field $\stackrel{30,31}{ }$, and the energy gap of the superconductor. Before discussing these phenomena, we will explain the way to get the current-density (sum of the paramagnetic currentdensity and antimagnetic one) within the present theory. The present CDFT can predict the transverse component of the paramagnetic current-density as well as the OPSS and electron density. Using the equation of continuity and taking into account the Helmholtz theorem for the vector analysis 71 , the longitudinal component of the paramagnetic current-density can be determined. Since the transverse component of the paramagnetic current-density is determined from the present CDFT directly, the whole component of the paramagnetic current-density can be obtained. On the other hand, the antimagnetic current-density can also be obtained from the present CDFT since it depends on the electron density that is one of the basic variables. Therefore, the sum of the paramagnetic current-density and antimagnetic one, i.e., current-density of the system, can be eventually obtained.

(B-1) The Meissner effect is generally investigated by calculating the magnetic field inside the superconducting system. The magnetic field does not exist in the superconducting phase except the boundary region where the current-density flows. The magnetic field is generally calculated from the current-density via the microscopic Maxwell equation $\stackrel{59,60}{ }$. Since the current-density can be obtained from the present CDFT, as mentioned above, it can be verified whether the magnetic field in the superconducting phase becomes zero or not. The present CDFT enables us to make the direct observation of the Meissner effect.

(B-2) Silsbee's rule (or the London equation) provides the relation between the critical magnetic field and critical current-density $\stackrel{30}{ }$. In the present CDFT, we can get the relation between the external magnetic field and the current-density existing when the OPSS disappears. That is to say, it can be argued to what extent the critical magnetic field and the corresponding current-density which are both calculated from the present CDFT are

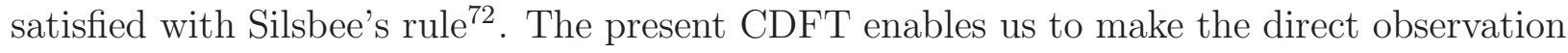
of Silsbee's rule as well as the Meissner effect.

(B-3) Besides the above phenomena, we can also discuss the energy gap of the superconductor by using the critical current-density. In general, the current-density flowing in the superconductor has the upper limit, and its magnitude is related to the energy gap. $\underline{22}$ Using 
the critical current-density calculated from the present CDFT, we can evaluate the magnitude of the energy gap of the superconductor. Comparing this result with the approximate solution $\left|D_{i}^{\text {singlet }}\right|$ or $\left|D_{i}^{\text {triplet }}\right|$ given in Sec. VI, it is possible to discuss to what extent the system is close to the BCS case.

Thus, the present CDFT for the superconductor would be effective for investigating the properties of the superconducting state which are related to the magnetic field and/or current-density flowing in the superconductor. 


\section{Appendix: Property of the BV transformation}

In this Appendix, we confirm that Eq. (44) can be regarded as a transformation which is similar to the unitary transformation ${ }^{73}$. Equation (44) is collectively written in a form of using matrices as

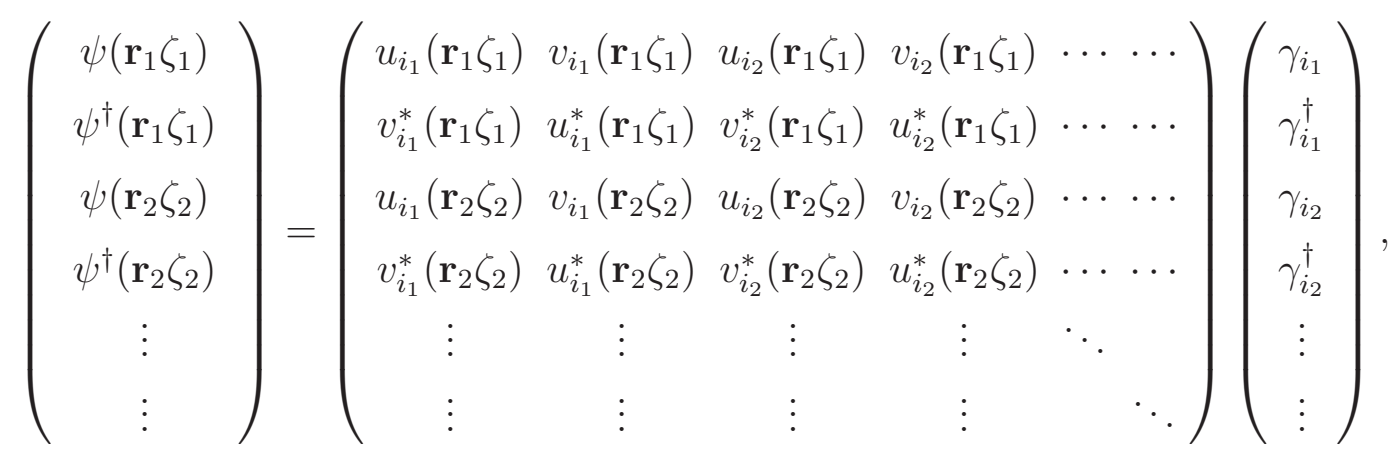

where $\mathbf{r}_{i}$ and $\mathbf{r}_{i+1}$ are, respectively, located in the infinitesimal volumes which are adjacent to each other. The infinitesimal volume is assumed to be $\delta^{3}$. Let us observe the property of the transformation matrix of Eq. A.1. If it is denoted as $\hat{U}$, then we have

$$
\hat{U}=\left(\begin{array}{ccccc}
\varpi_{i_{1}}\left(\mathbf{r}_{1} \zeta_{1}\right) & \varpi_{i_{2}}\left(\mathbf{r}_{1} \zeta_{1}\right) & \varpi_{i_{3}}\left(\mathbf{r}_{1} \zeta_{1}\right) & \ldots & \ldots \\
\varpi_{i_{1}}\left(\mathbf{r}_{2} \zeta_{2}\right) & \varpi_{i_{2}}\left(\mathbf{r}_{2} \zeta_{2}\right) & \varpi_{i_{3}}\left(\mathbf{r}_{2} \zeta_{2}\right) & \ldots & \ldots \\
\varpi_{i_{1}}\left(\mathbf{r}_{3} \zeta_{3}\right) & \varpi_{i_{2}}\left(\mathbf{r}_{3} \zeta_{3}\right) & \varpi_{i_{3}}\left(\mathbf{r}_{3} \zeta_{3}\right) & \ldots & \ldots \\
\vdots & \vdots & \vdots & \ddots & \\
\vdots & \vdots & \vdots & & \ddots
\end{array}\right),
$$

where $\varpi_{i_{\alpha}}\left(\mathbf{r}_{\beta} \zeta_{\beta}\right)$ is the constituent $2 \times 2$ block matrix defined as

$$
\varpi_{i_{\alpha}}\left(\mathbf{r}_{\beta} \zeta_{\beta}\right)=\left(\begin{array}{cc}
u_{i_{\alpha}}\left(\mathbf{r}_{\beta} \zeta_{\beta}\right) & v_{i_{\alpha}}\left(\mathbf{r}_{\beta} \zeta_{\beta}\right) \\
v_{i_{\alpha}}^{*}\left(\mathbf{r}_{\beta} \zeta_{\beta}\right) & u_{i_{\alpha}}^{*}\left(\mathbf{r}_{\beta} \zeta_{\beta}\right)
\end{array}\right)
$$

We shall consider the product $\hat{U}^{\dagger} \hat{U}$ :

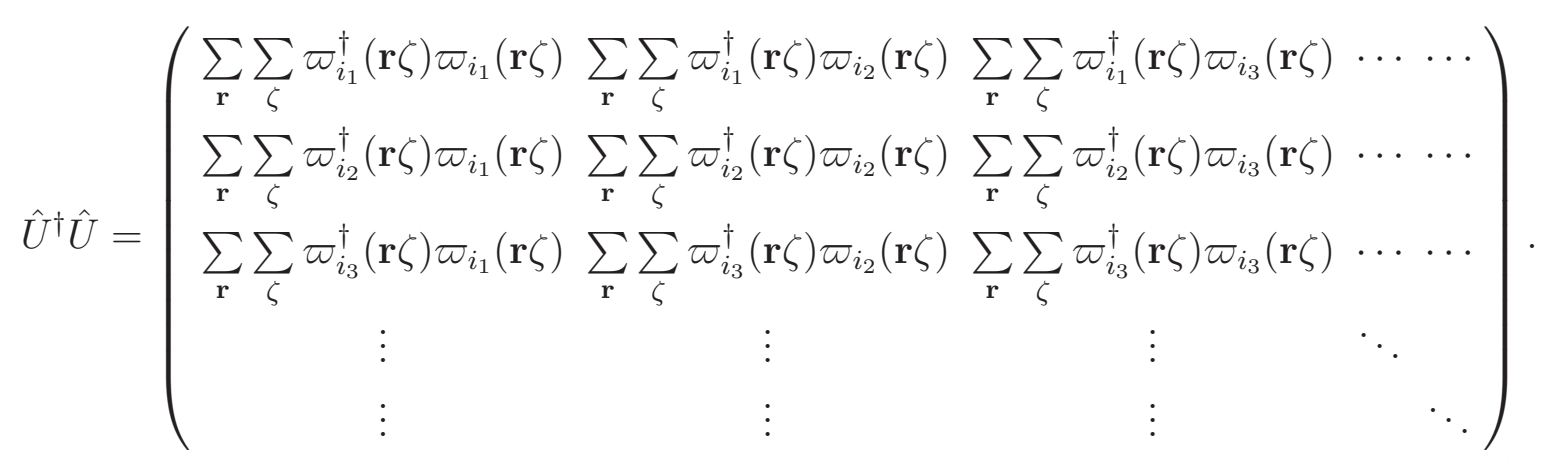


The diagonal $2 \times 2$ block matrix is written as

$$
\begin{gathered}
\sum_{\mathbf{r}} \sum_{\zeta} \varpi_{i_{\alpha}}^{\dagger}(\mathbf{r} \zeta) \varpi_{i_{\alpha}}(\mathbf{r} \zeta)=\sum_{\mathbf{r}} \sum_{\zeta}\left(\begin{array}{cc}
u_{i_{\alpha}}^{*}(\mathbf{r} \zeta) u_{i_{\alpha}}(\mathbf{r} \zeta) & u_{i_{\alpha}}^{*}(\mathbf{r} \zeta) v_{i_{\alpha}}(\mathbf{r} \zeta) \\
+v_{i_{\alpha}}(\mathbf{r} \zeta) v_{i_{\alpha}}^{*}(\mathbf{r} \zeta) & +v_{i_{\alpha}}(\mathbf{r} \zeta) u_{i_{\alpha}}^{*}(\mathbf{r} \zeta) \\
v_{i_{\alpha}}^{*}(\mathbf{r} \zeta) u_{i_{\alpha}}(\mathbf{r} \zeta) & v_{i_{\alpha}}^{*}(\mathbf{r} \zeta) v_{i_{\alpha}}(\mathbf{r} \zeta) \\
+u_{i_{\alpha}}(\mathbf{r} \zeta) v_{i_{\alpha}}^{*}(\mathbf{r} \zeta) & +u_{i_{\alpha}}(\mathbf{r} \zeta) u_{i_{\alpha}}^{*}(\mathbf{r} \zeta)
\end{array}\right) \\
=\frac{1}{\delta^{3}}\left(\begin{array}{ll}
1 & 0 \\
0 & 1
\end{array}\right),
\end{gathered}
$$

where we use Eqs. (62), (65), and the general relation $\sum_{\mathbf{r}_{i}} f\left(\mathbf{r}_{i}\right) \delta^{3}=\int f(\mathbf{r}) d^{3} r$. On the other hand, the off-diagonal $2 \times 2$ block matrix is written as

$$
\begin{aligned}
\sum_{\mathbf{r}} \sum_{\zeta} \varpi_{i_{\alpha}}^{\dagger}(\mathbf{r} \zeta) \varpi_{i_{\beta}}(\mathbf{r} \zeta)=\sum_{\mathbf{r}} \sum_{\zeta}\left(\begin{array}{cc}
u_{i_{\alpha}}^{*}(\mathbf{r} \zeta) u_{i_{\beta}}(\mathbf{r} \zeta) & u_{i_{\alpha}}^{*}(\mathbf{r} \zeta) v_{i_{\beta}}(\mathbf{r} \zeta) \\
+v_{i_{\alpha}}(\mathbf{r} \zeta) v_{i_{\beta}}^{*}(\mathbf{r} \zeta) & +v_{i_{\alpha}}(\mathbf{r} \zeta) u_{i_{\beta}}^{*}(\mathbf{r} \zeta) \\
v_{i_{\alpha}}^{*}(\mathbf{r} \zeta) u_{i_{\beta}}(\mathbf{r} \zeta) & v_{i_{\alpha}}^{*}(\mathbf{r} \zeta) v_{i_{\beta}}(\mathbf{r} \zeta) \\
+u_{i_{\alpha}}(\mathbf{r} \zeta) v_{i_{\beta}}^{*}(\mathbf{r} \zeta) & +u_{i_{\alpha}}(\mathbf{r} \zeta) u_{i_{\beta}}^{*}(\mathbf{r} \zeta)
\end{array}\right) \\
=\left(\begin{array}{ll}
0 & 0 \\
0 & 0
\end{array}\right),
\end{aligned}
$$

where we use Eqs. (62), (64) and the general relation mentioned above. Thus, Eqs. (A.5) and (A.6) lead to

$$
\hat{U}^{\dagger} \hat{U}=\frac{1}{\delta^{3}} \hat{I}
$$

where $\hat{I}$ is the unit matrix.

From Eq. (A.7), it can be shown that

$$
\hat{U} \hat{U}^{\dagger}=\frac{1}{\delta^{3}} \hat{I}
$$

We shall show the relations that are derived from Eq. (A.8). The product $\hat{U} \hat{U}^{\dagger}$ is given by

$$
\hat{U} \hat{U}^{\dagger}=\left(\begin{array}{ccccc}
\sum_{i} \varpi_{i}\left(\mathbf{r}_{1} \zeta_{1}\right) \varpi_{i}^{\dagger}\left(\mathbf{r}_{1} \zeta_{1}\right) & \sum_{i} \varpi_{i}\left(\mathbf{r}_{1} \zeta_{1}\right) \varpi_{i}^{\dagger}\left(\mathbf{r}_{2} \zeta_{2}\right) & \sum_{i} \varpi_{i}\left(\mathbf{r}_{1} \zeta_{1}\right) \varpi_{i}^{\dagger}\left(\mathbf{r}_{3} \zeta_{3}\right) & \cdots & \cdots \\
\sum_{i} \varpi_{i}\left(\mathbf{r}_{2} \zeta_{2}\right) \varpi_{i}^{\dagger}\left(\mathbf{r}_{1} \zeta_{1}\right) & \sum_{i} \varpi_{i}\left(\mathbf{r}_{2} \zeta_{2}\right) \varpi_{i}^{\dagger}\left(\mathbf{r}_{2} \zeta_{2}\right) & \sum_{i} \varpi_{i}\left(\mathbf{r}_{2} \zeta_{2}\right) \varpi_{i}^{\dagger}\left(\mathbf{r}_{3} \zeta_{3}\right) & \cdots & \ldots \\
\sum_{i} \varpi_{i}\left(\mathbf{r}_{3} \zeta_{3}\right) \varpi_{i}^{\dagger}\left(\mathbf{r}_{1} \zeta_{1}\right) & \sum_{i} \varpi_{i}\left(\mathbf{r}_{3} \zeta_{3}\right) \varpi_{i}^{\dagger}\left(\mathbf{r}_{2} \zeta_{2}\right) & \sum_{i} \varpi_{i}\left(\mathbf{r}_{3} \zeta_{3}\right) \varpi_{i}^{\dagger}\left(\mathbf{r}_{3} \zeta_{3}\right) & \cdots & \ldots \\
\vdots & \vdots & \vdots & \ddots & \\
\vdots & \vdots & \vdots &
\end{array}\right)
$$


The diagonal and off-diagonal $2 \times 2$ block matrices are written as

$$
\sum_{i} \varpi_{i}(\mathbf{r} \zeta) \varpi_{i}^{\dagger}(\mathbf{r} \zeta)=\sum_{i}\left(\begin{array}{cc}
u_{i}(\mathbf{r} \zeta) u_{i}^{*}(\mathbf{r} \zeta) & u_{i}(\mathbf{r} \zeta) v_{i}(\mathbf{r} \zeta) \\
+v_{i}(\mathbf{r} \zeta) v_{i}^{*}(\mathbf{r} \zeta) & +v_{i}(\mathbf{r} \zeta) u_{i}(\mathbf{r} \zeta) \\
v_{i}^{*}(\mathbf{r} \zeta) u_{i}^{*}(\mathbf{r} \zeta) & v_{i}^{*}(\mathbf{r} \zeta) v_{i}(\mathbf{r} \zeta) \\
+u_{i}^{*}(\mathbf{r} \zeta) v_{i}^{*}(\mathbf{r} \zeta) & +u_{i}^{*}(\mathbf{r} \zeta) u_{i}(\mathbf{r} \zeta)
\end{array}\right),
$$

and

$$
\sum_{i} \varpi_{i}\left(\mathbf{r}_{\alpha} \zeta_{\alpha}\right) \varpi_{i}^{\dagger}\left(\mathbf{r}_{\beta} \zeta_{\beta}\right)=\sum_{i}\left(\begin{array}{cc}
u_{i}\left(\mathbf{r}_{\alpha} \zeta_{\alpha}\right) u_{i}^{*}\left(\mathbf{r}_{\beta} \zeta_{\beta}\right) & u_{i}\left(\mathbf{r}_{\alpha} \zeta_{\alpha}\right) v_{i}\left(\mathbf{r}_{\beta} \zeta_{\beta}\right) \\
+v_{i}\left(\mathbf{r}_{\alpha} \zeta_{\alpha}\right) v_{i}^{*}\left(\mathbf{r}_{\beta} \zeta_{\beta}\right) & +v_{i}\left(\mathbf{r}_{\alpha} \zeta_{\alpha}\right) u_{i}\left(\mathbf{r}_{\beta} \zeta_{\beta}\right) \\
v_{i}^{*}\left(\mathbf{r}_{\alpha} \zeta_{\alpha}\right) u_{i}^{*}\left(\mathbf{r}_{\beta} \zeta_{\beta}\right) & v_{i}^{*}\left(\mathbf{r}_{\alpha} \zeta_{\alpha}\right) v_{i}\left(\mathbf{r}_{\beta} \zeta_{\beta}\right) \\
+u_{i}^{*}\left(\mathbf{r}_{\alpha} \zeta_{\alpha}\right) v_{i}^{*}\left(\mathbf{r}_{\beta} \zeta_{\beta}\right) & +u_{i}^{*}\left(\mathbf{r}_{\alpha} \zeta_{\alpha}\right) u_{i}\left(\mathbf{r}_{\beta} \zeta_{\beta}\right)
\end{array}\right),
$$

respectively. Compared these elements with Eq. (A.8), we have

$$
\begin{aligned}
& \sum_{i}\left\{u_{i}\left(\mathbf{r}_{\alpha} \zeta_{\alpha}\right) u_{i}^{*}\left(\mathbf{r}_{\beta} \zeta_{\beta}\right)+v_{i}\left(\mathbf{r}_{\alpha} \zeta_{\alpha}\right) v_{i}^{*}\left(\mathbf{r}_{\beta} \zeta_{\beta}\right)\right\}=\frac{1}{\delta^{3}} \delta_{\mathbf{r}_{\alpha} \mathbf{r}_{\beta}} \delta_{\zeta_{\alpha} \zeta_{\beta}}, \\
& \sum_{i}\left\{u_{i}\left(\mathbf{r}_{\alpha} \zeta_{\alpha}\right) v_{i}\left(\mathbf{r}_{\beta} \zeta_{\beta}\right)+v_{i}\left(\mathbf{r}_{\alpha} \zeta_{\alpha}\right) u_{i}\left(\mathbf{r}_{\beta} \zeta_{\beta}\right)\right\}=0 .
\end{aligned}
$$

Using the general relation $\frac{1}{\delta^{3}} \delta_{\mathbf{r}_{\alpha} \mathbf{r}_{\beta}} \delta_{\zeta_{\alpha} \zeta_{\beta}}=\delta\left(\mathbf{r}_{\alpha}-\mathbf{r}_{\beta}\right) \delta_{\zeta_{\alpha} \zeta_{\beta}}$, Eq. (A.12) exactly coincides with Eqs. (45) and (46). Thus, it is confirmed that the BV transformation owns the unitary-like property given by Eqs. (A.7) and (A.8).

\section{Acknowledgments}

This work was partially supported by Grant-in-Aid for Scientific Research (No. 26400354 and No. 26400397) of Japan Society for the Promotion of Science.

1 M. Lüders, M. A. L. Marques, N. N. Lathiotakis, A. Floris, G. Profeta, L. Fast, A. Continenza, S. Massidda, and E. K. U. Gross, Phys. Rev. B 72, 024545 (2005).

2 M. A. L. Marques, M. Lüders, N. N. Lathiotakis, G. Profeta, A. Floris, L. Fast, A. Continenza, E. K. U. Gross, and S. Massidda, Phys. Rev. B 72, 024546 (2005).

3 T. Kreibich and E. K. U. Gross, Phys. Rev. Lett. 86, 2984 (2001).

4 A. Floris, G. Profeta, N. N. Lathiotakis, M. Lüders, M. A. L. Marques, C. Franchini, E. K. U. Gross, A. Continenza, and S. Massidda, Phys. Rev. Lett. 94, 037004 (2005). 
5 G. Profeta, C. Franchini, N. N. Lathiotakis, A. Floris, A. Sanna, M. A. L. Marques, M. Lüders, S. Massidda, E. K. U. Gross, and A. Continenza, Phys. Rev. Lett. 96, 047003 (2006).

6 A. Sanna, C. Franchini, A. Floris, G. Profeta, N. N. Lathiotakis, M. Lüders, M. A. L. Marques, E. K. U. Gross, A. Continenza, and S. Massidda, Phys. Rev. B 73, 144512 (2006).

7 A. Floris, A. Sanna, S. Massidda, and E. K. U. Gross, Phys. Rev. B 75, 054508 (2007).

8 A. Sanna, G. Profeta, A. Floris, A. Marini, E. K. U. Gross, and S. Massidda, Phys. Rev. B 75, 020511(R) (2007).

9 P. Cudazzo, G. Profeta, A. Sanna, A. Floris, A. Continenza, S. Massidda, and E.K.U. Gross, Phys. Rev. Lett. 100, 257001 (2008); Phys. Rev. B 81, 134506 (2010).

10 J. Quintanilla, K. Capelle, and L. N. Oliveira, Phys. Rev. B 78, 205426 (2008).

11 C. Bersier, A. Floris, A. Sanna, G. Profeta, A. Continenza, E. K. U. Gross, and S. Massidda, Phys. Rev. B 79, 104503 (2009).

12 G. Stefanucci, E. Perfetto, and M. Cini, Phys. Rev. B 81, 115446 (2010).

13 R. Akashi, K. Nakamura, R. Arita, and M. Imada, Phys. Rev. B 86, 054513 (2012).

14 R. Akashi and R. Arita, Phys. Rev. Lett. 111, 057006 (2013); Phys. Rev. B 88, 014514 (2013).

15 K. Higuchi, K. Koide, T. Imanishi, and M. Higuchi, Int. J. Quantum Chem. 113, 709 (2013).

16 F. Essenberger, A. Sanna, A. Linscheid, F. Tandetzky, G. Profeta, P. Cudazzo, and E. K. U. Gross, Phys. Rev. B 90, 214504 (2014).

17 G. Csire, B. Újfalussy, J. Cserti, and B. Győrffy, Phys. Rev. B 91, 165142 (2015).

18 A. Linscheid, A. Sanna, F. Essenberger, and E. K. U. Gross, Phys. Rev. B 92, 024505 (2015).

19 A. Linscheid, A. Sanna, A. Floris, and E. K. U. Gross, Phys. Rev. Lett. 115, 097002 (2015).

20 F. Essenberger, A. Sanna, P. Buczek, A. Ernst, L. Sandratskii, and E. K. U. Gross, Phys. Rev. B 94, 014503 (2016).

21 J. A. Flores-Livas, A. Sannaa, and E. K. U. Gross, Eur. Phys. J. B 89, 63 (2016).

22 For instance, see, C. P. Poole Jr., et al., Superconductivity (Academic Press, London, 2007).

23 P. Hohenberg and W. Kohn, Phys. Rev. 136, B864 (1964).

24 W. Kohn and L. J. Sham, Phys. Rev. 140, A1133 (1965).

25 N. D. Mermin, Phys. Rev. 137, A1441 (1965).

26 L. N. Oliveira, E. K. U. Gross and W. Kohn, Phys. Rev. Lett 60, 2430 (1988).

27 W. Kohn, E. K. U. Gross and L. N. Oliveira, J. de Phyique (Paris) 50, 2601 (1989).

28 D. Larbalestier, in 100 Years of Superconductivity, edited by H. Rogalla and P. H. Kes (CRC 
Press, Florida, 2012), Chap 11.

29 W. Hassenzahl and O. Tsukamoto, in 100 Years of Superconductivity, edited by H. Rogalla and P. H. Kes (CRC Press, Florida, 2012), Chap 12.

30 M. Tinkham, Introduction to Superconductivity 2nd. Ed. (Dover Press, New York, 1996) Chap.2.

31 A. G. Rose-Innes and E. H. Rhoderick, Introduction to Superconductivity (Pergamon Press, New York, 1978) Chap. 7.

32 G. Vignale and M. Rasolt, Rhys. Rev. Lett. 59, 2360 (1987).

33 G. Vignale and M. Rasolt, Phys. Rev. B 37, 10685 (1988).

34 C. N. Yang, Rev. Mod. Phys. 34, 694 (1962).

35 A. J. Leggett, Quantum Liquids (Oxford University Press, Oxford, 2006).

36 M. Levy, Proc. Natl.Acad. Sci. U.S.A. 76, 6062 (1979).

37 M. Levy, Phys. Rev. A 26, 1200 (1982).

38 E. H. Lieb, Int. J. quantum Chem. 24, 243 (1983).

39 M. Higuchi and K. Higuchi, Phys. Rev. B 69, 035113 (2004).

40 K. Higuchi and M. Higuchi, Phys. Rev. B 69, 165118 (2004).

41 K. Higuchi and M. Higuchi, Phys. Rev. B 71, 035116 (2005).

42 K. Higuchi and M. Higuchi, Phys. Rev. A. 79, 022113 (2009).

43 M. Higuchi and K. Higuchi, Phys. Rev. A. 81, 042505 (2010).

44 R. M. Dreizler and E. K. U. Gross, Density Functional Theory (Springer-Verlag, Berlin, 1990).

45 K. Higuchi, K. Koide, T. Imanishi, and M. Higuchi, Int. J. Quantum Chem. 113, 709 (2013).

46 M. Higuchi and A. Hasegawa, J. Phys. Soc. Jpn 66, 149 (1997).

47 M. Higuchi and K. Higuchi, Phys. Rev. B 65, 195122 (2002).

48 P. G. de Gennes, Superconductivity of Metals and Alloys (W. A. Benjamin, New York, 1966) Chap. 5.

49 This type of eigenfunction of the RDM2 is called type (a) which was defined in our recent paper cited by Ref. 50 .

50 K. Higuchi, E. Miki and M. Higuchi, Cond-mat. arXiv:1512.07775.

51 J. E. Annett, Superconductivity, Superfluids and Condensates (Oxford University Press, Oxford, 2004) Chap. 5.

52 Chap. 3 in Ref. 44.

53 J. Bardeen, L. N. Cooper and J. R. Schrieffer, Phys. Rev. 108, 1175 (1957). 
54 J. R. Schrieffer, Theory of superconductivity (Westview Press, Colorado, 1999) Chap.2.

55 Chap. 3 in Ref. 30.

56 N. N. Bogoliubov, Nuovo Cimento 7, 794 (1958).

57 J. G. Valatin, Nuovo Cimento 7, 843 (1958).

58 For instance, see, S. Raimes, The Wave Mechanics of Electrons in Metals (North-Holland, New York, 1963).

59 J. D. Jackson, Classical Electrodynamics 3rd ed. (John Wiley \& Sons, New York, 1999) Chap. 6.

60 K. Cho, Reconstruction of Macroscopic Maxwell Equations (Springer-Verlag, Berlin, 2010) Chap. 1.

61 The current-density included in the microscopic Maxwell equation is the sum of the paramagnetic current-density and antimagnetic current-density. Concerning the paramagnetic currentdensity, the transverse component is obtained from the present CDFT directly, and the longitudinal component is calculated by using both the equation of continuity and Helmholtz theorem ${ }^{71}$. The antimagnetic current-density is also obtained from the present CDFT, because it depends on the electron density which is one of basic variables of this theory. The details will be discussed in Sec. VII.

62 O. Gunnarsson, M. Jonson, B. I. Lundqvist, Phys. Rev. B 20, 3136 (1979).

63 A similar expression can be found in the case of the conventional DFT at zero temperature ${ }^{23}, 24$, though the entropy terms do not appear in it. See, for example, Chap. 7 in Ref. 44.

64 G. F. Giuliani and G. Vignale, Quantum theory of the Electron Liquid (Cambridge Univ. Press, New York, 2005).

65 For instance, see, Phys. Chem. Chem. Phys. 16, 14333-14634 (2014).

66 As can be seen in Eq. (136), the case when the effective pair potential disappears corresponds to $V_{0}=0$ which means the nonexistence of the attractive interaction in the Hamiltonian Eq. (5). Then, the system is necessarily in the normal state.

67 K. Higuchi and M. Higuchi, Phys. Rev. B 74, 195122 (2006); Phys. Rev. B 75, 159902(E) (2007).

68 K. Higuchi and M. Higuchi, J. Phys.: Condens. Matter 19, 365216 (2007).

69 M. Higuchi and K. Higuchi, Phys. Rev. B 75, 195114 (2007).

70 K. Higuchi and M. Higuchi, J. Phys. Soc. Jpn. 80, SA123 (2011). 
71 G. B. Arfken and H. J. Weber, Mathematical Methods for Physicists (Elsevier Academic Press, Massachusetts, 2005) Chap. 1.

72 For instance, see, Chap. 8.4 in Ref. 31.

73 In some textbooks, e.g., Ref. 48, this transformation is explicitly called the unitary transformation. 